\title{
The Gaia-ESO Survey: Abundance ratios in the inner-disk open clusters Trumpler 20, NGC 4815, NGC 6705^
}

\author{
L. Magrini ${ }^{1}$, S. Randich ${ }^{1}$, D. Romano ${ }^{2}$, E. Friel ${ }^{3}$, A. Bragaglia ${ }^{2}$, R. Smiljanic ${ }^{4,5}$, H. Jacobson ${ }^{6}$, A. Vallenari ${ }^{7}$, M. Tosi ${ }^{2}$, \\ L. Spina ${ }^{1,8}$, P. Donati ${ }^{2,9}$, E. Maiorca ${ }^{1}$, T. Cantat-Gaudin ${ }^{7,10}$, R. Sordo ${ }^{7}$, M. Bergemann ${ }^{11}$, F. Damiani ${ }^{12}$, G. \\ Tautvaišienè $^{13}$, S. Blanco-Cuaresma ${ }^{14,15}$, F. Jiménez-Esteban ${ }^{16}$, D. Geisler ${ }^{17}$, N. Mowlavi1 ${ }^{18}$, C. Munoz ${ }^{17}$, I. San

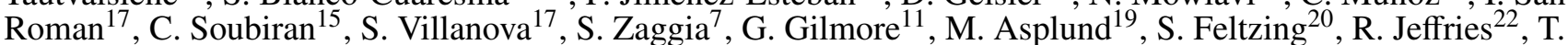 \\ Bensby $^{20}$, P. Francois ${ }^{21}$, S. Koposov ${ }^{11,23}$, A. J. Korn ${ }^{20}$, E. Flaccomio ${ }^{12}$, E. Pancino ${ }^{2,24}$, A. Recio-Blanco ${ }^{25}$, G. Sacco ${ }^{1}$, \\ M. T. Costado $^{26}$, E. Franciosini ${ }^{1}$, P. Jofre ${ }^{11}$, P. de Laverny ${ }^{25}$, V. Hill ${ }^{25}$, U. Heiter ${ }^{27}$, A. Hourihane ${ }^{11}$, R. Jackson ${ }^{22}$, C.

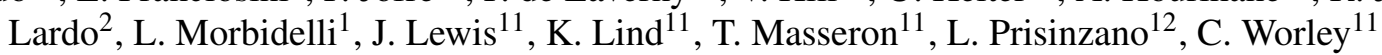

INAF-Osservatorio Astrofisico di Arcetri, Largo E. Fermi, 5, I-50125 Firenze, Italy e-mail: laura@arcetri.astro.it

INAF-Osservatorio Astronomico di Bologna, Via Ranzani, 1, 40127, Bologna, Italy

Department of Astronomy, Indiana University, Bloomington, USA

${ }^{4}$ Department for Astrophysics, Nicolaus Copernicus Astronomical Center, ul. Rabiańska 8, 87-100 Toruń, Poland

5 European Southern Observatory, Karl-Schwarzschild-Str. 2, 85748 Garching bei München, Germany

${ }^{6}$ MIT Kavli Institute, Boston, USA

Osservatorio Astronomico di Padova, Vicolo dell'Osservatorio, 5, 35122, Padova, Italy

8 Dipartimento di Fisica, sezione di Astronomia, Largo E. Fermi, 2, I-50125 Firenze, Italy

9 Dipartimento di Fisica e Astronomia, Via Ranzani, 1, 40127, Bologna, Italy

10 Dipartimento di Fisica e Astronomia, Vicolo dell'Osservatorio, 3, 35122, Padova, Italy

11 Institute of Astronomy, University of Cambridge, Madingley Road, Cambridge, CB3 OHA, UK

12 Osservatorio Astronomico di Palermo, P.zza del Parlamento 1, 90134, Palermo, Italy

13 Institute of Theoretical Physics and Astronomy, Vilnius University, A. Gostauto 12, 01108 Vilnius, Lithuania

${ }^{14}$ Univ. Bordeaux, LAB, UMR 5804, F-33270, Floirac, France.

15 CNRS, LAB, UMR 5804, F-33270, Floirac, France

16 Centro de Astrobiología (INTA-CSIC) P.O. Box 78, 28691 Villanueva de la Canada, Madrid, Spain

17 Departamento de Astronoma, Universidad de Concepción, Casilla 160-C, Concepción, Chile

18 Astronomy Department, University of Geneva, Ch. des Maillettes 51, 1290 Versoix, Switzerland

19 Research School of Astronomy and Astrophysics, Australian National University, Canberra, ACT 2611, Australia

20 Lund Observatory, Department of Astronomy and Theoretical Physics, Box 43, SE-221 00 Lund, Sweden

21 Observatoire de Paris, CNRS, Univ. Paris Diderot, place Jules Janssen, 92190, Meudon, France

22 Astrophysics Group, Keele University, Keele, Staffordshire, ST5 5BG, UK

23 Moscow M.V. Lomonosov State University, Sternberg Astronomical Institute, Universitetskij pr., 13, 119992 Moscow, Russia

24 ASI Science Data Center, I-00044 Frascati, Italy

25 Laboratoire Lagrange (UMR7293), Universit de Nice Sophia Antipolis, CNRS, Observatoire de la Cte dAzur, BP 4229,F-06304 Nice cedex 4, France

${ }^{26}$ Instituto de Astrofísica de Andalucía (IAA-CSIC), Glorieta de la Astronomía, E-18008-Granada, Spain

27 Department of Physics and Astronomy, Uppsala University, Box 516, 75120 Uppsala, Sweden

Received ; accepted

\section{ABSTRACT}

Context. Open clusters are key tools to study the spatial distribution of abundances in the disk and their evolution with time. Aims. Using the first release of stellar parameters and abundances of the Gaia-ESO Survey, we analyse the chemical properties of stars in three old/intermediate-age open clusters, namely NGC 6705, NGC 4815, and Trumpler 20, all located in the inner part of the Galactic disk at Galactocentric radius $\mathrm{R}_{G C} \sim 7 \mathrm{kpc}$, aiming at proving their homogeneity and at comparing them with the field population.

Methods. We study the abundance ratios of elements belonging to two different nucleosynthetic channels: $\alpha$-elements and ironpeak elements. For each element we analyse the internal chemical homogeneity of cluster members and we compare the cumulative distributions of cluster abundance ratios with those of solar neighbourhood turn-off stars and of inner-disk/bulge giants. We compare the abundance ratios of field and cluster stars with two chemical evolution models that predict different $\alpha$-enhancement dependences on the Galactocentric distance due to different assumptions on the infall and star formation rates.

Results. The main results can be summarised as follows: i) cluster members are chemically homogeneous within 3- $\sigma$ in all analysed elements; ii) the three clusters have comparable $[\mathrm{El} / \mathrm{Fe}]$ patters within $\sim 1-\sigma$, but they differ in their global metal content $[\mathrm{El} / \mathrm{H}]$, with NGC 4815 having the lowest metallicity. Their [El/Fe] ratios show differences and analogies with those of the field population, both in the solar neighbourhood and in the bulge/inner disk; iii) comparing the abundance ratios with the results of two chemical evolution models and with field star abundance distributions, we find that the abundance ratios of $\mathrm{Mg}, \mathrm{Ni}, \mathrm{Ca}$ in NGC 6705 might require an inner birthplace, implying a subsequent variation of its $\mathrm{R}_{G C}$ during its lifetime, consistent with previous orbit determination.

Conclusions. Using the results of the first internal data release, we show the potential of the Gaia-ESO Survey, through a homogeneous and detailed analysis of the cluster versus field populations, to reveal the chemical structure of our Galaxy using a completely uniform analysis of different populations. We verify that the Gaia-ESO Survey data are able to identify the unique chemical properties of each cluster, pinpointing the composition of the interstellar medium at the epoch and place of formation. The full dataset of the Gaia-ESO Survey will be a superlative tool to constrain the chemical evolution of our Galaxy by disentangling different formation and evolution scenarios. 


\section{Introduction}

Open clusters are very useful tracers of the processes of formation and evolution of our Galaxy. They are a disc population located from the inner parts of the disk to its outskirts, and with ages spanning from few Myr for recently formed clusters to several Gyr for old clusters (see, e.g., Dias et al., 2002; Dias et al., 2012). The population of young open clusters has a small scale height above the Galactic plane $(\sim 60 \mathrm{pc})$, while old open clusters reach higher altitudes $\sim 350 \mathrm{pc}$ (Chen et al. 2003), thus being all presumably part of the thin component of the disk. Kinematics of open clusters, both in terms of rotation and velocity dispersions, are also consistent with association with the thin disk of the Galaxy (Scott et al. 1995, Wu et al. 2009). In addition, also form a chemical point of view, the roughly solar abundance ratios of open clusters further supports to an association with the thin disk.

Their ages and distances can be derived from colourmagnitude diagrams, obtained via photometric studies, making them a perfect instrument to investigate the temporal changes in the spatial distribution of abundances (e.g., Magrini et al. 2009, Yong et al., 2012).

In the framework of the study of cluster population, the GaiaESO Survey (Gilmore et al. 2012, Randich \& Gilmore 2012), together with other spectroscopic surveys observing open clusters, such as the Apache Point Observatory Galactic Evolution Experiment (APOGEE, Allende-Prieto et al. 2008), is allowing us to study a large number of young, intermediate-age, and old open clusters. Within the APOGEE project, the Open Cluster Chemical Analysis and Mapping (OCCAM) survey aims to produce a comprehensive, uniform, infrared-based data set for hundreds of open clusters, and constrain key Galactic dynamical and chemical parameters from this sample. A first contribution to the OCCAM project has been recently published by Frinchaboy et al. (2013) that presented the analysis of 141 members stars in 28 open clusters. This new dataset allowed them to revise the Galactic metallicity gradient.

In particular, old and intermediate-age clusters are rare fossils of the past star formation history of the Galactic disk. Apparently, the old open clusters observed at present time with ages over $\sim 1$ Gyr have survived because of their peculiar initial characteristics, such as their larger than average mass, higher central concentration, and orbits that allow them to avoid the disruptive influence of the giant molecular clouds (Friel 1995 Janes \& Phelps 1994; Bonatto et al. 2006). This might introduce possible differences in chemical composition between field and cluster stars presently observed at the same Galactocentric radius, since both might have moved from their place of birth, but in different ways.

The majority of stars born in open clusters were indeed dispersed into the Galaxy field in a relatively short time, i.e., within the first Gyr from their formation (see, e.g., Janes \& Phelps 1994, Lada \& Lada 2003, Gieles et al. 2006). This phenomenon is more rapid in the inner disk where the density of stars is higher (Freeman 1970, van der Kruit 2002). Thus the existence of several old and intermediate-age open clusters within the Solar circle $\left(\mathrm{R}_{\mathrm{GC}}<8 \mathrm{kpc}\right)$, such as those observed during the first periods of observation of the Gaia-ESO Survey, offers a unique oppor-

\section{Send offprint requests to: L. Magrini}

* Based on observations collected with the FLAMES spectrograph at VLT/UT2 telescope (Paranal Observatory, ESO, Chile), for the GaiaESO Large Public Survey (188.B-3002). tunity to study the evolution of the disk in a region so far little explored.

Specifically, this study focuses on detailed and homogeneously obtained chemical abundance patterns of different populations, that offer valuable clues in the interpretation of the Galactic history. We refer to the paper of Jacobson et al. (in prep.) for a discussion of the spatial distribution of the global metallicity and its implications for the radial Galactic metallicity gradient.

As a first approximation, we expect the abundances of open clusters to match those of the field stars at similar Galactocentric distances, but observations are revealing differences (see, e.g., Yong et al. 2005, 2012; De Silva et al. 2007) and these differences contain important information about, e.g., the place where the open clusters were born, the homogeneity of the disk at any $\mathrm{R}_{\mathrm{GC}}$ at the epoch when the cluster formed, etc..

More in details, studies that compare field and cluster populations were tackled by several authors in the past. For example, De Silva et al. (2007) analysed the chemical pattern of the inner old open cluster Collinder 261, comparing its abundance ratios with those of Cepheid stars and of field stars. They found remarkable differences for some elements, such as $\mathrm{Na}, \mathrm{Mg}, \mathrm{Si}$, and $\mathrm{Ba}$. They claimed that the differences are a signature of the local inhomogeneities at the time and site of cluster formation. Other examples can be found in the works of Yong et al. (2005, 2012) and of Sestito et al. (2008), who studied open clusters located at $\mathrm{R}_{\mathrm{GC}}>13 \mathrm{kpc}$. They compared the chemical properties of several outer disk open clusters with those of stellar clusters located in the disk within $\mathrm{R}_{\mathrm{GC}}<13 \mathrm{kpc}$, with other stellar tracers located in the outer disk, such as red giant stars, Cepheids, and the solar neighbourhood stars. They found that the behaviour of $\alpha$-elements is not exactly the same in all their clusters, but that it is on average similar to that of solar neighbourhood stars. They concluded that the primary difference between solar neighbourhood and outer disk is that the chemical enrichment in the outer disk did not yet reach the metallicities of the solar neighbourhood, but the contribution of the two nucleosynthetic channels, SNII and SNIa, appear to have been similar. However, the conclusions of these works are usually based on heterogeneous samples including literature and authors' own results. Heterogeneous samples might mask genuine abundance differences and/or artificially induce (or amplify) abundance differences between otherwise chemically similar populations. The different effects are driven by the size of the samples and by the elements considered.

In this framework the Gaia-ESO Survey data, with its uniform data-set and its homogeneous analysis, will allow, for the first time, a comparison of different populations on a footing not possible before.

As an initial step in this direction, the first data release of the Gaia-ESO Survey, including the first six months of observations, allows us to analyse in detail the chemical composition of three old and intermediate-age open clusters located in the very inner disk (NGC 6705, NGC 4815, and Trumpler 20), and to compare them with the field population in the solar neighbourhood and with evolved stars located in the inner-disk/bulge.

The present paper is structured as follows: In Sec. 2 we briefly describe the Gaia-ESO Survey. In Sec. 3 we present the properties and membership of the first three old/intermediateage clusters observed by the Gaia-ESO Survey. In Sec. 4 we summarise the target selection strategy for field stars. In Sec. 5 we check the quality of the analysis of cluster stars and we anal- 
yse the abundance patterns of the three open cluster and compare them to the field population, whereas in Sec. 6 we speculate on the origin of their abundance ratios. In Sec. 7 we give our summary.

\section{The Gaia-ESO Survey and its first data release}

The Gaia-ESO Survey is a large, public spectroscopic survey started at the end of 2011 that is employing the VLT FLAMES (Pasquini et al. 2002) instrument to obtain high quality spectroscopy of $\sim 10^{5}$ stars in our Galaxy. The observed stars belong to well defined samples and are selected making use of several photometric databases such as the VISTA Hemisphere Survey (VHS) (McMahon 2012), the Two Micron All Sky Survey (2MASS, Skrutskie et al. 2006) and a variety of photometric surveys of open clusters. The focus of the Gaia-ESO Survey is to quantify the kinematical and chemical element abundance distributions in the different components of the Milky Way: bulge, thin and thick disks, halo, and about a hundred open clusters spanning a large range of ages, distances, and masses. A general description of the Survey can be found in Gilmore et al. (in prep.) and Randich et al. (in prep.).

In the present work we discuss results of the analysis of UVES (Dekker et al. 2000) spectra of F-G-K stars. This analysis is described in details in Smiljanic et al. (in prep.). We briefly review how recommended parameters are computed. The recommended parameters are obtained combining the results of different nodes considering first the accuracy of each node judged using a sample of calibration stars with well-known stellar parameters, called benchmark stars, as reference (see Jofre et al. $(2013 \mathrm{a}$ b $)$ ). Among the benchmark stars, there are stars having stellar parameters comparable to that of the stars discussed in the present paper, as, e.g., $\xi$ Hya.

The different approaches of the nodes con be summarised as follows: $i$ ) nodes that employ the equivalent width (EW) analysis, obtaining EWs form the observed spectra. The atmospheric parameter determination is based on the excitation and ionisation balance of iron lines; ii) spectrum synthesis methods that derive the atmospheric parameters from a $\chi^{2}$ fit to observed spectra. In some cases the computation of EWs from best-matching synthetic spectra is used to derive the individual element abundances; iii) multi-linear regression methods that simultaneously determine the stellar parameters of an observed spectrum by the projection of the spectrum onto vector functions, constructed as an optimal linear combination of the local synthetic spectra.

The nodes using the EW method are eight, while the remaining five nodes adopt different approaches to the spectral synthesis. Details on the individual node techniques are in Smiljanic et al. (in prep.).

Further consistency tests are conducted using the calibration clusters and other calibration targets, such as, e.g., the globular clusters NGC1851 and NGC2808, for which the nodes' and recommended parameters were compared with PARSEC isochrones in the $\mathrm{T}_{e f f}$ vs. log g plane finding a good agreement, and the CoRoT (COnvection ROtation and planetary Transits, Baglin et al. 2006) giant stars. Then the parts of the parameter space where a given node under-performs are identified 1

Finally, the median value of the validated results is adopted as the recommended value of that parameter. For the cluster stars

\footnotetext{
1 Nodes that cannot analyse the benchmark stars and reproduce their atmospheric parameters $\left(\mathrm{T}_{e f f}\right.$ and $\left.\log \mathrm{g}\right)$ within $150 \mathrm{~K}$ and $0.30 \mathrm{dex}$, respectively, are disregarded.
}

discussed in the present paper, the recommended values are typically derived using the results of 8-10 nodes. For the solar neighbourhood stars and the inner-disk/bulge stars the parameters are, on average, determined using the results of nine nodes.

The uncertainties are taken to be the method-to-method dispersions. Once the recommended values of the atmospheric parameters of all stars are defined, the spectroscopic analysis proceeds to its second step, the determination of elemental abundances. The nodes use the recommended parameters to recompute elemental abundances, and the median values of the resulting abundances are the final recommended best values.

We emphasise here that cluster and field stars are analysed in a completely homogeneous way. Parameters and abundances for stars observed during the first six months have been delivered within the Gaia-ESO Survey consortium in an internal Data Release (GESviDR1Final), which includes abundances of FeI, FeII, NiI, CrI, TiI, TiII, SiI, CaI, MgI, NaI, AlI, ZnI, YII, ZrII, CeII. Unfortunately, the abundances of neutron capture elements are available only for a small sub-sample of stars, and thus they are not discussed in the present paper. The recommended values are used in the rest of the paper. In particular, we use the abundances derived with the recommended parameters, including those of iron (computed considering only Fe I lines). The Gaia-ESO survey abundances are scaled to the solar abundances of Grevesse et al. (2007). The errors on [Fe/H] are comparable among the different samples of solar-neighbourhood stars, inner disk stars, and intermediate-age cluster stars discussed in the present paper, with cool stars having in general higher errors both in cluster and Milky Way stars. Typical errors are of the order of $0.1 \mathrm{dex}$, and they become $\sim 0.2$ dex for several stars cooler than $\sim 4500 \mathrm{~K}$.

\section{The old and intermediate-age clusters in DR1}

Three old and intermediate-age open clusters were observed and analysed in DR 1: NGC 6705, NGC4815, and Trumpler 20. They are all located within the Solar circle in a region still poorly investigated but of great importance for our understanding of the mechanisms of disk/bulge formation. Being close to the Galactic centre (see Table 1), the three clusters might suffer from strong tidal effects, as well as frequent interactions with molecular clouds, and thus they can provide important constraints to the cluster survival in a 'hostile' environment (see, e.g., Janes \& Phelps 1994; Lada \& Lada 2003; Gieles et al. 2006). They might also probe a key issue to understand the mechanism of Galaxy formation and evolution, i.e., the radial metallicity gradient, which was the subject of a number of studies in past decades using open clusters as tracers (see, e.g., Janes 1979; Panagia \& Tosi 1981; Friel 1995; Twarog et al. 1997; Carraro et al. 1998; Bragaglia \& Tosi 2006; Sestito et al.|2006, 2007, 2008, Magrini et al. 2009; Pancino et al.|2010; Andreuzzi et al.|2011; Jacobson et al. 2011). The first results from the Gaia-ESO Survey are helping to shed light on the radial metallicity gradient in the very inner part of the disk, a region still poorly investigated (Jacobson et al. in prep.). Stars observed in the three clusters were selected on the basis of their colour-magnitude diagrams: targets for GIRAFFE were mainly main-sequence stars, while targets for UVES were evolved stars belonging to the red clump. More details on the target selection can be found in Bragaglia et al. (in prep.) and the papers on individual clusters, as, e.g., Donati et al. (in prep.) for Trumpler 20 and Friel et al. (in prep.) for NGC 4815. 
In the present paper, we show the results of $[\mathrm{Fe} / \mathrm{H}]$ and abundance ratios of member stars of the three clusters presented above and observed with UVES. The membership has been derived using the information on the radial velocities measured in the Gaia-ESO Survey spectra in the paper of Donati et al. (in prep) for Trumpler 20, in Friel et al. (in prep.) for NGC 4815, and in Cantat-Gaudin et al. (in prep.) for NGC 6705. Briefly, the radial velocities are firstly used to identify the systemic velocity of the cluster, and then to remove stars beyond a certain $\sigma$ level from the median velocity to separate cluster member stars from the field.

The cluster parameters are summarised in Table 1, where we show the names of the clusters, their coordinates (equatorial and Galactic), the reddening $\mathrm{E}(\mathrm{B}-\mathrm{V})$, the age in Gyr, the turnoff (TO) mass in solar masses, the distance from the Sun, the Galactocentric distance, and the height from the Galactic plane in $\mathrm{kpc}$, the metallicity, $[\mathrm{Fe} / \mathrm{H}]$, and the number of members analysed. For a discussion and comparison with literature values of the three clusters we refer to the papers of Donati et al. (2013), Friel et al. (in prep.), and Cantat-Gaudin et al. (in prep.).

\section{UVES observations of field stars in DR1}

To compare the abundance ratios of the different populations of the Milky Way and of open clusters in a fully homogeneous way, we have considered the results in GESviDR1Final for the Milky Way field stars. These stars belong to two samples: the solar neighbourhood sample and the bulge/inner disk sample. For a complete description about how these samples are defined we refer to Gilmore et al. (in prep.).

The solar neighbourhood sample The observation criteria for the UVES stars aim at including the three major solar neighbourhood population groups (halo, thick disk, old thin disk). They are designed to obtain an unbiased sample of $\sim 5000 \mathrm{G}$-stars within $2 \mathrm{kpc}$ from the Sun. The purpose of this sample is to quantify in detail the local elemental abundance distribution functions. The sample used for the present work includes 390 stars, and corresponds to the Milky Way turn-off (TO) stars with recommended parameters in GESviDR1Final.

The bulge and inner disk The UVES stars observed together with the GIRAFFE stars dedicated to the study of Galactic bulge are expected to be evolved stars belonging to both bulge and inner disk populations. The prime targets of the GIRAFFE observations are $\mathrm{K}$ giants, including the red clump stars, with typical magnitude $I=15$. The brighter $\mathrm{K}$ giant stars in the same fields are the targets of UVES, and they allow sampling bulge and inner Galaxy populations. During the first six months of the GaiaESO Survey, 32 stars of the bulge/inner-disk were observed and are included in the present discussion.

\section{The abundance patterns of open clusters}

The first step of our analysis is thus to check if the abundance ratios in each clusters are independent of the stellar parameters. To derive the abundance ratios we use the iron abundance computed by the nodes with the recommended stellar parameters. In Table 2 we present the ids, the radial velocities, the recommended values of stellar parameters $\left(\mathrm{T}_{e f f}, \log \mathrm{g}\right.$ and $\left.\xi\right)$ with their errors and the elemental abundance with their error (expressed in the logarithmic form $\mathrm{A}(\mathrm{El})=12+\log (\mathrm{El} / \mathrm{H})$ ) for members in open clusters. As anticipated in Sec. 2, the uncertainties on the stellar parameters $\left(\mathrm{T}_{e f f}, \log \mathrm{g}, \xi\right.$ and $\left.[\mathrm{Fe} / \mathrm{H}]\right)$ in $\mathrm{DR} 1$ are the method-to-method dispersions. For Fe I two errors are given: the method-to-method dispersion $(\sigma$ (FeI), in column 6$)$ computed in the calculation of the recommended atmospheric parameters, and the node-to-node dispersion in $\mathrm{A}(\mathrm{FeI})$ of abundances re-computed with the recommended parameters (the error on $[\mathrm{Fe} / \mathrm{H}]$ presented in column 5 ). 
Table 1. Clusters' parameters.

\begin{tabular}{lllllllllllr}
\hline \hline Name & $\alpha$ & $\begin{array}{c}\delta \\
\mathrm{J} 2000.0\end{array}$ & $\begin{array}{l}\mathrm{l} \\
(\mathrm{deg})\end{array}$ & $\begin{array}{l}\mathrm{b} \\
(\mathrm{deg})\end{array}$ & $\mathrm{E}(\mathrm{B}-\mathrm{V})$ & $\begin{array}{l}\text { Age } \\
(\mathrm{Gyr})\end{array}$ & $\begin{array}{l}\text { TO mass } \\
\left(\mathrm{M}_{\odot}\right)\end{array}$ & $\begin{array}{l}\mathrm{D}_{\odot} \\
(\mathrm{kpc})\end{array}$ & $\begin{array}{l}\mathrm{R}_{G C}{ }^{d} \\
(\mathrm{kpc})\end{array}$ & {$[\mathrm{Fe} / \mathrm{H}]$} & N.of members \\
\hline \hline NGC6705 $^{a}$ & $18: 51: 05$ & $-06: 16: 12$ & 27.31 & -2.78 & 0.43 & $0.30 \pm 0.05$ & $\sim 3$ & 1.9 & 6.3 & $+0.14 \pm 0.06$ & 21 \\
Trumpler20 $^{b}$ & $12: 39: 32$ & $-60: 37: 36$ & 301.48 & 2.22 & 0.33 & $1.50 \pm 0.15$ & $\sim 1.8$ & 2.4 & 6.88 & $+0.17 \pm 0.05$ & 13 \\
NGC4815 $^{c}$ & $12: 57: 59$ & $-64: 57: 36$ & 303.63 & -2.10 & 0.72 & $0.57 \pm 0.07$ & $\sim 2.5$ & 2.5 & 6.9 & $+0.03 \pm 0.05$ & 5 \\
\hline \hline
\end{tabular}

$a$ - NGC 6705 parameters from Cantat-Gaudin et al. (in prep.). $b$ - Trumpler 20 parameters from Donati et al. (in prep.). $c-$ NGC 4815 parameters from Friel et al. (in prep.). $d$ - computed with $\mathrm{R}_{\odot}=8 \mathrm{kpc}$. 
Table 2. Stellar parameters and abundance for member stars.

\begin{tabular}{|c|c|c|c|c|c|c|c|c|c|c|c|c|}
\hline 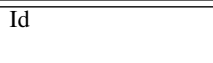 & $\begin{array}{l}\mathrm{T}_{\text {eff }} \\
\mathrm{K}\end{array}$ & $\overline{l \log g}$ & $\begin{array}{ll}\xi \\
\mathrm{km} \mathrm{s}^{-1}\end{array}$ & 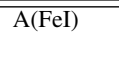 & $\overline{c \sigma(\mathrm{FeI})}$ & $\overline{\mathrm{A}(\mathrm{FeII})}$ & $\begin{array}{l}\mathrm{A}(\mathrm{MgI}) \\
\end{array}$ & 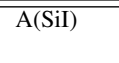 & $\overline{\mathrm{A}(\mathrm{CaI})}$ & $\begin{array}{ll}\mathrm{A}(\mathrm{TiI}) \\
\end{array}$ & 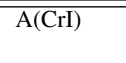 & "A(NiI) \\
\hline 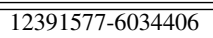 & $4849 \pm 41$ & $2.86 \pm 0.13$ & $\begin{array}{l}1.27 \pm 0.11 \\
\end{array}$ & $7.50 \pm 0.03$ & 0.06 & $7.46 \pm 0.05$ & $\begin{array}{l}7.67 \pm 0.09 \\
\end{array}$ & $7.54 \pm 0.05$ & 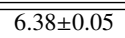 & $4.91 \pm 0.02$ & $5.66 \pm 0.07$ & $6.18 \pm 0.03$ \\
\hline $12392585-6038279$ & $5034 \pm 106$ & $3.12 \pm 0.31$ & $1.25 \pm 0.1$ & $7.66 \pm 0.01$ & 0.08 & $7.64 \pm 0.07$ & $7.79 \pm 0.06$ & $7.70 \pm 0.04$ & $6.46 \pm 0.01$ & $5.04 \pm 0.02$ & $5.81 \pm 0.03$ & $6.37 \pm 0.01$ \\
\hline $12392700-6036053$ & $4800 \pm 77$ & $2.80 \pm 0.24$ & $1.29 \pm 0.1$ & $7.60 \pm 0.03$ & 0.07 & $7.57 \pm 0.04$ & $7.72 \pm 0.02$ & $7.63 \pm 0.06$ & $6.38 \pm 0.04$ & $4.91 \pm 0.03$ & $5.67 \pm 0.02$ & $6.34 \pm 0.04$ \\
\hline $12393132-6039422$ & $4954 \pm 64$ & $3.07 \pm 0.15$ & $1.20 \pm 0.22$ & $7.64 \pm 0.02$ & 0.05 & $7.67 \pm 0.06$ & $7.75 \pm 0.02$ & $7.68 \pm 0.04$ & $6.47 \pm 0.05$ & $5.01 \pm 0.03$ & $5.76 \pm 0.06$ & $6.37 \pm 0.02$ \\
\hline $12393782-6039051$ & $4909 \pm 129$ & $2.80 \pm 0.21$ & $1.30 \pm 0.16$ & $7.61 \pm 0.04$ & 0.12 & $7.62 \pm 0.06$ & $7.75 \pm 0.06$ & $7.66 \pm 0.04$ & $6.42 \pm 0.04$ & $4.97 \pm 0.01$ & $5.71 \pm 0.02$ & $6.32 \pm 0.02$ \\
\hline $12394419-6034412$ & $4941 \pm 90$ & $2.88 \pm 0.23$ & $1.25 \pm 0.06$ & $7.66 \pm 0.04$ & 0.07 & $7.67 \pm 0.03$ & $7.75 \pm 0.04$ & $7.64 \pm 0.05$ & $6.50 \pm 0.03$ & $5.03 \pm 0.02$ & $5.76 \pm 0.03$ & $6.38 \pm 0.01$ \\
\hline $12394475-6038339$ & $4850 \pm 112$ & $2.75 \pm 0.22$ & $1.38 \pm 0.08$ & $7.58 \pm 0.02$ & 0.05 & $7.62 \pm 0.06$ & $7.75 \pm 0.04$ & $7.66 \pm 0.02$ & $6.39 \pm 0.02$ & $4.93 \pm 0.04$ & $5.71 \pm 0.04$ & $6.32 \pm 0.02$ \\
\hline $12394596-6038389$ & $4912 \pm 118$ & $2.87 \pm 0.21$ & $1.27 \pm 0.15$ & $7.63 \pm 0.03$ & 0.06 & $7.64 \pm 0.04$ & $7.72 \pm 0.05$ & $7.63 \pm 0.04$ & $6.44 \pm 0.07$ & $4.94 \pm 0.02$ & $5.71 \pm 0.01$ & $6.34 \pm 0.04$ \\
\hline $12394690-6033540$ & $4968 \pm 77$ & $3.03 \pm 0.1$ & $1.14 \pm 0.14$ & $7.70 \pm 0.04$ & 0.11 & $7.69 \pm 0.04$ & $7.79 \pm 0.04$ & $7.70 \pm 0.05$ & $6.47 \pm 0.04$ & $5.01 \pm 0.01$ & $5.76 \pm 0.03$ & $6.42 \pm 0.02$ \\
\hline $12394742-6038411$ & $4900 \pm 100$ & $2.73 \pm 0.23$ & $1.21 \pm 0.15$ & $7.62 \pm 0.04$ & 0.05 & $7.59 \pm 0.02$ & $7.75 \pm 0.04$ & $7.70 \pm 0.02$ & $6.43 \pm 0.04$ & $4.94 \pm 0.01$ & $5.73 \pm 0.03$ & $6.32 \pm 0.03$ \\
\hline $12395426-6038369$ & $4925 \pm 100$ & $2.98 \pm 0.13$ & $1.36 \pm 0.04$ & $7.63 \pm 0.02$ & 0.07 & $7.66 \pm 0.08$ & $7.74 \pm 0.05$ & $7.67 \pm 0.04$ & $6.42 \pm 0.03$ & $5.01 \pm 0.02$ & $5.72 \pm 0.06$ & $6.37 \pm 0.02$ \\
\hline $12395975-6035072$ & $4850 \pm 87$ & $2.79 \pm 0.19$ & $1.29 \pm 0.11$ & $7.62 \pm 0.03$ & 0.07 & $7.61 \pm 0.08$ & $7.74 \pm 0.06$ & $7.67 \pm 0.03$ & $6.42 \pm 0.03$ & $4.92 \pm 0.03$ & $5.69 \pm 0.03$ & $6.31 \pm 0.02$ \\
\hline $12400278-6041192$ & $4932 \pm 67$ & $2.98 \pm 0.11$ & $1.37 \pm 0.05$ & $7.58 \pm 0.02$ & 0.10 & $7.61 \pm 0.07$ & $7.71 \pm 0.07$ & $7.66 \pm 0.03$ & $6.42 \pm 0.03$ & $4.96 \pm 0.03$ & $5.71 \pm 0.07$ & $6.32 \pm 0.04$ \\
\hline $12572442-6455173$ & $4198 \pm 88$ & $1.56 \pm 0.37$ & $1.37 \pm 0.05$ & $7.48 \pm 0.03$ & 0.0 & $7.53 \pm 0.03$ & $7.85 \pm 0.04$ & $7.6 \pm 0.1$ & $6.23 \pm 0.07$ & $4.66 \pm 0.03$ & $5.5 \pm 0.01$ & \pm 0.04 \\
\hline $12574328-6457386$ & $4895 \pm 40$ & $2.40 \pm 0.13$ & $1.73 \pm 0.14$ & $7.45 \pm 0.02$ & 0.08 & $7.46 \pm 0.05$ & $7.68 \pm 0.1$ & $7.47 \pm 0.05$ & $6.29 \pm 0.05$ & $4.75 \pm 0.03$ & $5.54 \pm 0.03$ & $6.13 \pm 0.04$ \\
\hline $12575511-6458483$ & $4870 \pm 84$ & $2.55 \pm 0.47$ & $1.43 \pm 0.24$ & $7.51 \pm 0.01$ & 0.07 & $7.55 \pm 0.06$ & $7.78 \pm 0.07$ & $7.5 \pm 0.01$ & $6.32 \pm 0.02$ & $4.8 \pm 0.03$ & $5.56 \pm 0.07$ & $6.18 \pm 0.08$ \\
\hline $12575529-6456536$ & $5068 \pm 73$ & $2.79 \pm 0.25$ & $1.15 \pm 0.12$ & & 0.1 & & & & & & & \\
\hline $12580262-6456492$ & $4926 \pm 77$ & $2.57 \pm 0.08$ & $1.53 \pm 0.14$ & $7.56 \pm 0.02$ & 0.06 & $7.55 \pm 0.04$ & $7.69 \pm 0.17$ & $7.62 \pm 0.04$ & $6.37 \pm 0.05$ & $4.91 \pm 0.02$ & $5.6 \pm 0.05$ & $6.23 \pm 0.01$ \\
\hline $18503724-0614364$ & $4820 \pm 71$ & $2.42 \pm 0.21$ & $1.82 \pm 0.13$ & $7.47 \pm 0.02$ & 0.14 & $7.54 \pm 0.08$ & $7.78 \pm 0.09$ & $7.62 \pm 0.05$ & $6.3 \pm 0.07$ & $4.83 \pm 0.0$ & $5.62 \pm 0.04$ & $6.21 \pm 0.07$ \\
\hline $18504737-0617184$ & $4325 \pm 130$ & $1.72 \pm 0.29$ & $1.56 \pm 0.16$ & $7.54 \pm 0.04$ & 0.15 & $7.54 \pm 0.16$ & $7.85 \pm 0.03$ & $7.63 \pm 0.08$ & $6.35 \pm 0.05$ & $4.87 \pm 0.05$ & $5.64 \pm 0.04$ & $6.3 \pm 0.01$ \\
\hline 18505494-0616182 & $4689 \pm 109$ & & $1.46 \pm$ & & 0. & & & & & & & \\
\hline $18505581-0618148$ & $4577 \pm 139$ & $2.23 \pm 0.31$ & $1.60 \pm 0.24$ & $7.69 \pm 0.03$ & 0.18 & $7.61 \pm 0.08$ & $7.89 \pm 0.08$ & $7.8 \pm 0.05$ & $6.49 \pm 0.02$ & $5.16 \pm 0.04$ & $5.8 \pm 0.09$ & $6.49 \pm 0.01$ \\
\hline $18505755-0613461$ & $4873 \pm 114$ & $2.37 \pm 0.32$ & $1.33 \pm 0.19$ & $7.53 \pm 0.04$ & 0.1 & $7.38 \pm 0.01$ & $7.71 \pm 0.04$ & $7.66 \pm 0.04$ & $6.46 \pm 0.1$ & $4.87 \pm 0.07$ & $5.59 \pm 0.03$ & $6.21 \pm 0.15$ \\
\hline $18505944-0612435$ & $4925 \pm 177$ & $2.56 \pm 0.39$ & $1.50 \pm 0.5$ & $7.66 \pm 0.05$ & 0. & $7.68 \pm 0.07$ & 0.12 & $7.68 \pm 0.06$ & $6.42 \pm 0.12$ & .04 & $5.79 \pm 0.07$ & $6.4 \pm 0.05$ \\
\hline $18510023-0616594$ & $4433 \pm 95$ & $1.94 \pm 0.47$ & $1.50 \pm 0.14$ & $7.63 \pm$ & 0.12 & $7.58 \pm 0.13$ & $7.91 \pm 0.1$ & $7.71 \pm 0.13$ & $6.42 \pm 0.02$ & $5.05=$ & $5.75 \pm 0.07$ & $6.45 \pm 0.01$ \\
\hline $18510032-0617183$ & $4850 \pm 100$ & $2.38 \pm 0.21$ & $1.60 \pm 0.33$ & $7.55 \pm 0.03$ & 0.1 & $7.55 \pm 0.09$ & $7.84 \pm 0.1$ & $7.63 \pm 0.06$ & $6.43 \pm 0.08$ & $4.94 \pm 0.03$ & $5.68 \pm 0.02$ & $6.31 \pm 0.04$ \\
\hline 18510200-0617265 & $4415 \pm 87$ & $2.35 \pm 0.45$ & $1.48 \pm 0.07$ & $7.7 \pm 0.02$ & 0.1 & $7.82 \pm 0.1$ & $7.94 \pm 0.08$ & $7.78 \pm 0.06$ & $6.48 \pm 0.01$ & $5.03 \pm 0.02$ & $5.7 \pm 0.02$ & $6.47 \pm 0.02$ \\
\hline 18510289-0615301 & $4750 \pm 112$ & $2.40 \pm 0.28$ & $1.45 \pm 0.13$ & $7.55 \pm 0.01$ & 0. & $7.53 \pm 0.08$ & $7.76 \pm 0.11$ & $7.66 \pm 0.06$ & $6.44 \pm 0.06$ & $5.02 \pm 0.02$ & $5.73 \pm 0.06$ & $6.32 \pm 0.04$ \\
\hline $18510341-0616202$ & $4975 \pm 146$ & $2.50 \pm 0.3$ & $1.94 \pm 0.27$ & $7.59 \pm 0.07$ & 0. & $7.46 \pm 0.07$ & $7.97 \pm 0.06$ & $7.58 \pm 0.07$ & $6.42 \pm 0.07$ & $5.15 \pm 0.02$ & $5.75 \pm 0.06$ & $6.31 \pm 0.0$ \\
\hline $18510358-0616112$ & $4832 \pm 79$ & $2.31 \pm 0.31$ & $1.62 \pm 0.19$ & $7.59 \pm 0.01$ & 0 & $7.62 \pm 0.11$ & $7.79 \pm 0.06$ & $7.72 \pm 0.08$ & $6.44 \pm 0.01$ & $4.98=$ & $5.68 \pm 0.02$ & $6.36 \pm 0.02$ \\
\hline $18510786-0617119$ & $4768 \pm 53$ & 2. & $1.80 \pm 0.28$ & 7.5 & 0. & 0.04 & 8. & 7. & 6.4 & 01 & 0.06 & $6.29 \pm 0.03$ \\
\hline 18510833-0616532 & $4750 \pm 112$ & $2.25 \pm$ & $1.60 \pm 0.25$ & $7.66 \pm($ & 0.1 & $7.62 \pm 0.04$ & $7.93 \pm 0.1$ & $7.66 \pm 0.07$ & $6.45 \pm 0.02$ & $4.96 \pm 0.02$ & $5.78 \pm 0.12$ & $6.44 \pm 0.02$ \\
\hline $18511013-0615486$ & $4439 \pm 59$ & $1.87 \pm 0.53$ & $1.50 \pm 0.1$ & $7.53 \pm 0.02$ & 0.12 & $7.51 \pm 0.09$ & $7.84 \pm 0.1$ & $7.66 \pm 0.08$ & $6.35 \pm 0.01$ & $4.82 \pm 0.05$ & $5.6 \pm 0.03$ & $6.34 \pm 0.04$ \\
\hline $18511048-0615470$ & $4744 \pm 122$ & & $1.70 \pm$ & $7.55 \pm 0.05$ & 0. & $7.53=$ & $8.02 \pm 0.03$ & 7.58 & 6.41 & $4.98 \pm 0.04$ & 5.71 & $6.31 \pm 0.01$ \\
\hline $18511452-0616551$ & $4800 \pm 59$ & $2.40 \pm 0$ & $1.69 \pm$ & $7.6 \pm 1$ & 0. & $7.63 \pm 0$ & $7.83 \pm$ & $7.72 \pm 0.08$ & $6.48 \pm 0.07$ & $5.03 \pm 0.04$ & $5.72 \pm 0.03$ & $6.39 \pm 0.02$ \\
\hline 18511534-0618359 & $4755 \pm 57$ & $2.16 \pm 0.21$ & $1.79 \pm 0.17$ & $7.58 \pm 0.02$ & 0.22 & $7.53 \pm 0.01$ & $7.99 \pm 0.09$ & $7.72 \pm 0.1$ & $6.45 \pm 0.03$ & $5.03 \pm 0.04$ & $5.77 \pm 0.04$ & $6.36 \pm 0.03$ \\
\hline 18511571-0618146 & $4710 \pm 159$ & $2.27 \pm 0.3$ & $1.60 \pm 0.18$ & $7.64 \pm 0.03$ & 0.12 & $7.66 \pm 0.04$ & $7.81 \pm 0.12$ & $7.7 \pm 0.04$ & $6.45 \pm 0.04$ & $5.0 \pm 0.02$ & $5.74 \pm 0.04$ & $6.43 \pm 0.02$ \\
\hline $18512662-0614537$ & $4459 \pm 91$ & $2.10 \pm 0.48$ & $1.48 \pm 0.19$ & $7.61 \pm 0.01$ & 0.17 & & $7.93 \pm 0.14$ & $7.8 \pm 0.14$ & $6.45 \pm 0.02$ & & $5.75 \pm 0.06$ & $6.44 \pm 0.01$ \\
\hline $18514130-0620125$ & $4671 \pm 140$ & $2.20 \pm 0.28$ & $62 \pm 0.2$ & $7.61 \pm 0.03$ & 0.19 & $7.55 \pm 0.09$ & $7.8 \pm 0.1$ & $7.68 \pm 0.06$ & $6.41 \pm 0.0$ & $4.93 \pm 0.01$ & $5.74 \pm 0.04$ & $6.37 \pm 0.01$ \\
\hline
\end{tabular}



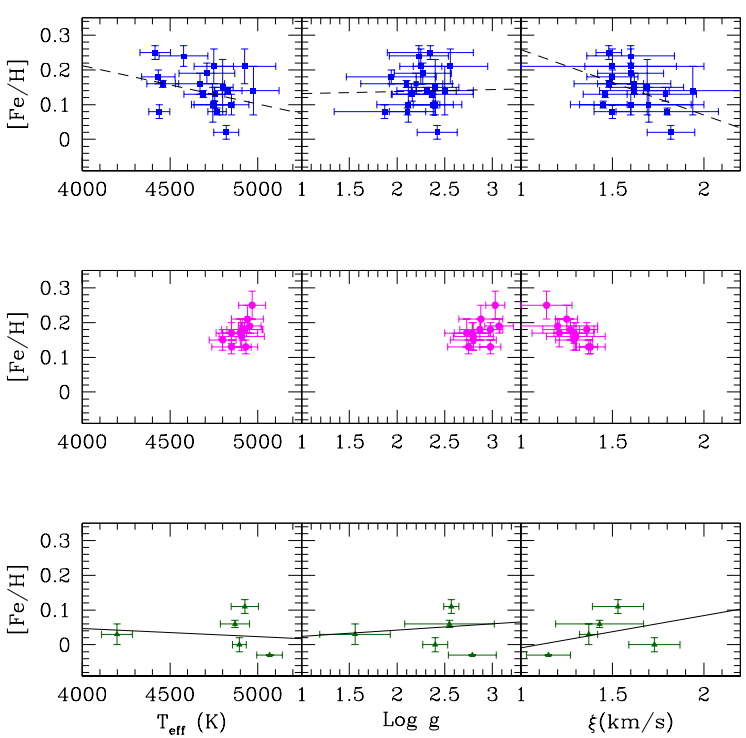

Fig. 1. $[\mathrm{Fe} / \mathrm{H}]$ versus stellar parameters in the three clusters. The results of NGC 6705 (blue) are shown in the upper panels, while Trumpler 20 (magenta) is in the middle panels, and NGC 4815 (dark green) in the bottom panels.

The results are shown in Fig. 1 for $[\mathrm{Fe} / \mathrm{H}]$ and in Figs. 2 for [El/Fe]. In Fig. 1 we plot the stellar parameters versus $[\mathrm{Fe} / \mathrm{H}]$ for member stars in the three clusters. We show also the mean least squares fits to the data for NGC 6705 and NGC 4815. We do not plot the linear fits for Trumpler 20 , since they are artificially driven by the small interval spanned in stellar parameters by its member stars. The trends are almost absent for NGC 4815, while some trends are present for NGC 6705, even if the ranges in $\mathrm{T}_{e f f}$, $\log \mathrm{g}$ and $\xi$ spanned by its members are again quite small.

In Figs. 2 the plots of $[\mathrm{El} / \mathrm{Fe}]$ versus stellar parameters are shown. The trends with $\mathrm{T}_{\text {eff }}$ and $\log \mathrm{g}$ are almost zero for all elements for NGC 6705 and Trumpler 20 where we have greater statistics, with a possible exception of $[\mathrm{Mg} / \mathrm{Fe}]$. The trends with $\xi$ seem to be more important, and might affect more elements. In general the cluster stars do not show important trends which could affect our further analysis.

\subsection{Confirming the chemical homogeneity of clusters}

It is often stated that open clusters are among the best objects for tracing the star-formation history in the disk (see, e.g., Friel 1995). They are considered to be composed of simple stellar populations, i.e., homogeneous in terms of age and chemical composition. Thus, we investigate the degree of homogeneity of the abundances of cluster members. We compare the standard

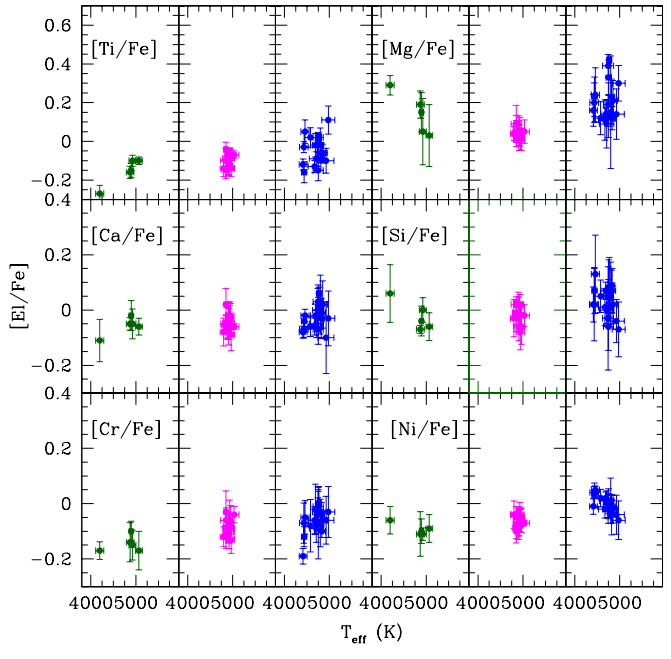

(a) Temperature

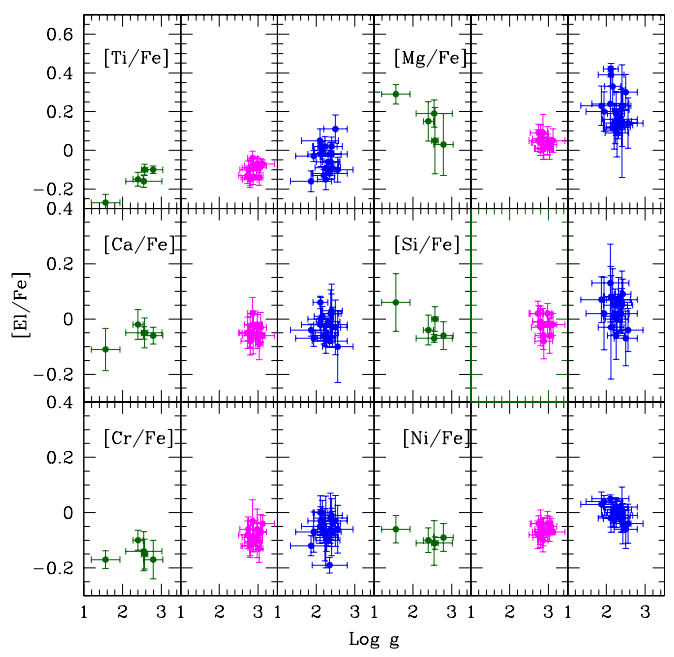

(b) Gravity

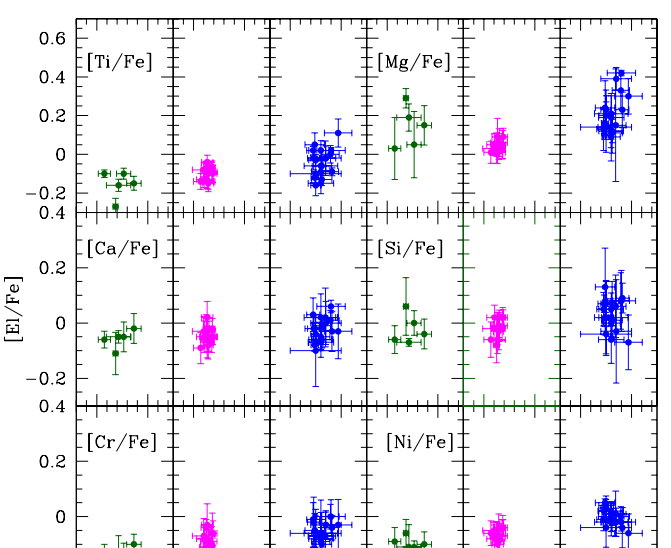


deviation $(\sigma)$ of all elements with the average uncertainty in the abundances $(\Delta=<\delta>)$, that is computed averaging the error on abundance ratios of each member star $(\delta)$. We considered the cluster homogeneous in a specified element if the intrinsic scatter, as given by the $\sigma$, is lower or comparable to the average error $\Delta$, which should be indicative of the expected dispersion.

The results are shown in Table 3 and in Figs. 3, 4, 5. The rectangles indicate the regions $1-\sigma$ wide around the average. The three clusters are essentially homogeneous in all elements. For Trumpler 20 we notice the presence of a star (\#123915776034406) that is slightly metal poorer than the main body of cluster members. However, its radial velocity and stellar parameters are in agreement with those of the other observed clump stars. As discussed in Donati et al. (2013), the Besançon model (Robin et al. 2003) computed at the location of Trumpler 20 gives that indicatively $17 \%$ of the candidate members for radial velocities may be still field stars. This corresponds to $\sim 1-2$ stars being possibly non-members in a sample of 13 stars, and it could justify the lower [Fe/H] of \#12391577-6034406. For NGC 6705 we note a possible bi-modal behaviour of $[\mathrm{Mg} / \mathrm{Fe}]$ with four stars outside the 1- $\sigma$ rectangle around the average (see also the trends in Figs. 2).

In Table 3 we report for each cluster: the abundance ratios, the standard deviation $\sigma$ and the average error on each measurement, $\Delta$, and the number of stars used to compute these quantities. For $[\mathrm{El} / \mathrm{Fe}]$ ratios $\Delta$ take into account the errors on $[\mathrm{El} / \mathrm{H}]$ and on $[\mathrm{Fe} / \mathrm{H}]$, summed in quadrature. We note, however, that this approach might overestimate the error on $[\mathrm{El} / \mathrm{Fe}]$ since the error on $[\mathrm{El} / \mathrm{H}]$ and $[\mathrm{Fe} / \mathrm{H}]$ are likely correlated. The best approach should be to consider the effect on $[\mathrm{El} / \mathrm{Fe}]$ due to the errors on the atmospheric parameters, but this kind of errors are not included in the present release. For the error on $[\mathrm{Fe} / \mathrm{H}]$ we used the values in column $\sigma(\mathrm{Fe} \mathrm{I})$ of Table 2. If we consider the best determined element, i.e. iron, we note that $\sigma$ is lower than $\Delta$. This is true for most of the other elements, with the possible exception of $\mathrm{Mg}$ and $\mathrm{Ti}$ which have a larger scatter in NGC 6705 and NGC 4815. Thus we can conclude that, with the present level of precision, these open clusters are homogeneous with respect to their content in $\alpha$-elements ( $\mathrm{Ti}, \mathrm{Si}, \mathrm{Ca}, \mathrm{Mg}$ ) and iron-peak elements (Fe, Ni, Cr). In Figs. 3, 4, 5 these results are reinforced: the abundance ratios of clusters do not show any correlation with $[\mathrm{Fe} / \mathrm{H}]$, and within the errors, they are homogeneous.

\subsection{Chemical patterns}

Having established the chemical homogeneity of these elements in the three clusters under analysis, we can compare their abundance patterns using the average abundances as representative of the entire cluster. In Fig. 6, we present graphically these results. An important feature is the comparison of the average abundance ratios in the three open clusters. As said in the previous sections, Trumpler 20, NGC4815, and NGC 6705 are located at similar distances from the Galactic Centre. They mainly differ in terms of age, cluster mass, and metallicity $[\mathrm{Fe} / \mathrm{H}]$. In terms of $[\mathrm{Fe} / \mathrm{H}]$, the metal poorest cluster (NGC 4815) and the metal richest one (Trumpler 20) differ $\sim 2-\sigma$.

Inspecting Fig. 6, we note that each cluster shows unique features with respect to the other clusters: Trumpler 20 has solar $[\mathrm{Si} / \mathrm{Fe}],[\mathrm{Mg} / \mathrm{Fe}]$ and $[\mathrm{Ca} / \mathrm{Fe}]$, slightly depleted in $[\mathrm{Ti} / \mathrm{Fe}]$, $[\mathrm{Ni} / \mathrm{Fe}]$ and $[\mathrm{Cr} / \mathrm{Fe}]$. NGC 4815 is solar in $[\mathrm{Si} / \mathrm{Fe}]$ and $[\mathrm{Ca} / \mathrm{Fe}]$ and slightly depleted $[\mathrm{Ti} / \mathrm{Fe}],[\mathrm{Cr} / \mathrm{Fe}]$ and $[\mathrm{Ni} / \mathrm{Fe}]$, and enhanced
Table 3. $\sigma$ and $\Delta$ for abundance ratios in each cluster.

\begin{tabular}{|c|c|c|c|}
\hline El & Mean & $\overline{\sigma^{(a)}}$ & $\overline{\Delta^{(b)}}$ \\
\hline \multicolumn{4}{|l|}{ 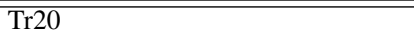 } \\
\hline \multicolumn{4}{|c|}{$N($ number of member stars $)=13$} \\
\hline$[\mathrm{Fe} / \mathrm{H}]$ & 0.17 & 0.05 & 0.07 \\
\hline$[\mathrm{Si} / \mathrm{Fe}]$ & -0.02 & & 0.08 \\
\hline$[\mathrm{Ca} / \mathrm{Fe}]$ & -0.05 & & 0.08 \\
\hline$[\mathrm{Mg} / \mathrm{l}$ & 04 & & 0.09 \\
\hline$[\mathrm{Ti} / \mathrm{Fe}$ & 10 & & 0.08 \\
\hline$[\mathrm{Ni} / \mathrm{Fe}]$ & -0.06 & & 0.08 \\
\hline$[\mathrm{Cr} / \mathrm{Fe}]$ & -0.08 & & 0.08 \\
\hline \multirow{2}{*}{\multicolumn{4}{|c|}{$\begin{array}{l}\text { NGC } 4815 \\
N \text { (number of member stars) }=5\end{array}$}} \\
\hline & & & \\
\hline$[\mathrm{Fe} / \mathrm{H}]$ & 0.03 & 0.05 & 0.10 \\
\hline$[\mathrm{Si} / \mathrm{Fe}]$ & -0.02 & & 0.09 \\
\hline$[\mathrm{Ca} / \mathrm{Fe}]$ & -0.06 & & 0.09 \\
\hline$[\mathrm{Mg} / \mathrm{F}$ & 0.14 & & 0.13 \\
\hline$[\mathrm{Ti} / \mathrm{Fe}]^{(a)}$ & -0.16 & 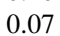 & 0.08 \\
\hline$[\mathrm{Ni} / \mathrm{Fe}]$ & -0.09 & 0.02 & 0.09 \\
\hline$[\mathrm{Cr} / \mathrm{Fe}]$ & -0.15 & 0.03 & 0.09 \\
\hline \multirow{2}{*}{\multicolumn{4}{|c|}{$\begin{array}{l}\text { NGC } 6705 \\
\mathrm{~N} \text { (number of member stars) }=21\end{array}$}} \\
\hline & & & \\
\hline$[\mathrm{Fe} / \mathrm{H}]$ & 0.14 & 0.06 & 0.14 \\
\hline ICi & 0.03 & & 0.1 \\
\hline$[\mathrm{Ca} / \mathrm{Fe}]$ & -0.02 & 0.05 & 0.14 \\
\hline$[\mathrm{Mg} / \mathrm{Fe}]$ & 0.20 & 0.09 & 0.17 \\
\hline$[\mathrm{Ti} / \mathrm{Fe}]^{(a)}$ & -0.05 & . & 0. \\
\hline$[\mathrm{Ni} / \mathrm{Fe}]$ & 0.01 & & 0.1 \\
\hline$[\mathrm{Cr} / \mathrm{Fe}]$ & -0.07 & 0.05 & 0.15 \\
\hline
\end{tabular}

(a) $\sigma$ : standard deviation of all elements; (b) $\Delta$ : the average uncertainty in the abundances $(\Delta=<\delta>)$

(c) $[\mathrm{Ti} / \mathrm{Fe}]$ is computed using only Ti I lines.

in $[\mathrm{Mg} / \mathrm{Fe}]$. NGC 6705 has solar $[\mathrm{Ti} / \mathrm{Fe}],[\mathrm{Si} / \mathrm{Fe}],[\mathrm{Ca} / \mathrm{Fe}]$ and $[\mathrm{Ni} / \mathrm{Fe}]$, while it is enhanced in $[\mathrm{Mg} / \mathrm{Fe}]$ and depleted in $[\mathrm{Cr} / \mathrm{Fe}]$. We statistically quantified the level of significance of the clusterto-cluster abundance differences estimating, for each abundance ratio in each pair of clusters, the following quantity

$$
\left.\left([\mathrm{El} / \mathrm{Fe}]_{\text {cluster } 1}-[\mathrm{El} / \mathrm{Fe}]_{\text {cluster } 2}\right) / \sqrt{(} \delta\left([\mathrm{El} / \mathrm{Fe}]_{\text {cluster1 }}\right)^{2}+\delta\left([\mathrm{El} / \mathrm{Fe}]_{\text {cluster } 2}\right)^{2}\right),
$$

where the abundance ratios and their errors are those reported in Table 3 All pairs of comparison are of the order of $1-\sigma$, with the lowest difference for $[\mathrm{Si} / \mathrm{Fe}]$ in Trumpler 20 and NGC 4815, and the largest difference for $[\mathrm{Ni} / \mathrm{Fe}]$ in NGC 6705 and NGC 4815 that differ more than 2- $\sigma$. This suggests that, in terms of abundance ratios over iron, these clusters are statistically very similar, but they differ in their global content of metals. We have thus done the same comparison with the elemental abundances, $[\mathrm{El} / \mathrm{H}]$. The results are shown in Fig 7, from which we see how the abundances of NGC 4815 differ from those of Trumpler 20 and NGC 6705 for all elements. We recomputed the level of significance of the cluster-to-cluster abundance differences finding that NGC 4815 differs $\sim 2-\sigma$ from the other two clusters in most of its $[\mathrm{El} / \mathrm{H}]$ abundances.

These differences, especially those of $[\mathrm{El} / \mathrm{H}]$, can be considered intrinsic characteristics of the chemical composition of the interstellar medium (ISM) from which each cluster was born since the analysis was performed in a fully homogeneous way, from the target selection, to the observational strategy, data reduction, and abundance analysis. A tentative first conclusion might be that, even if at present Trumpler 20, NGC 4815 and NGC 6705 are located at similar distances from the Galactic 


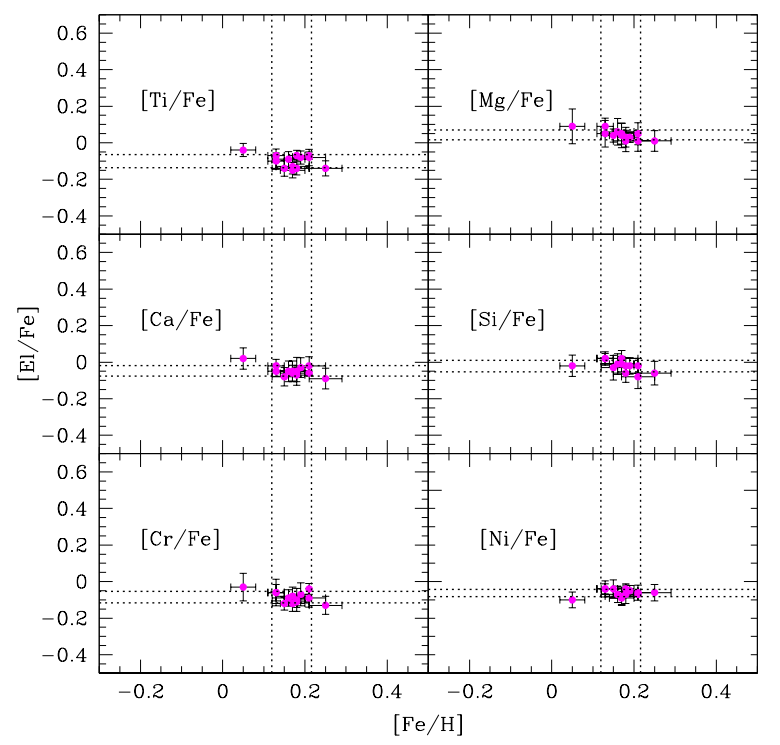

Fig. 3. Abundance ratios versus $[\mathrm{Fe} / \mathrm{H}]$ for individual member stars in $\mathrm{Tr} 20$. Errors on abundance ratios $[\mathrm{El} / \mathrm{Fe}]$ are computed summing in quadrature the errors in $[\mathrm{El} / \mathrm{H}]$ and the errors on $[\mathrm{Fe} / \mathrm{H}]$. In each panel, the rectangular region shown by intersection of the four dotted lines indicate the 1- $\sigma$ area around the average value.

Centre (GC) (although NGC 6705 is on the opposite side of the Sun-Galactic Centre line), they did not originate from an ISM with the same composition. In particular the difference in their mean metallicity is relevant, NGC 4815 having a lower metallicity (within 2- $\sigma$ ) than the other two clusters. This can be obtained, at least, in three different ways: $i$ ) the ISM is not azimuthally homogeneous, and areas located at similar radii might have a different chemical composition due to local enrichment and to incomplete mixing; $i$ ) the clusters might have moved from their place of birth, and thus they might reflect the chemical composition at a different radius with respect to their present position; iii) due to the different ages of the clusters, their mean metallicity and abundance ratios might be a signature of the temporal chemical evolution of the Galactic disk. A comparison with the field population, in the approximation that the migration does not dominate its distribution, is useful to check these hypotheses and in Sec 6 we describe a comparison with two different Galactic chemical evolution models to search for a possible explanation about the origin of the abundance ratios in these inner-disk open clusters.

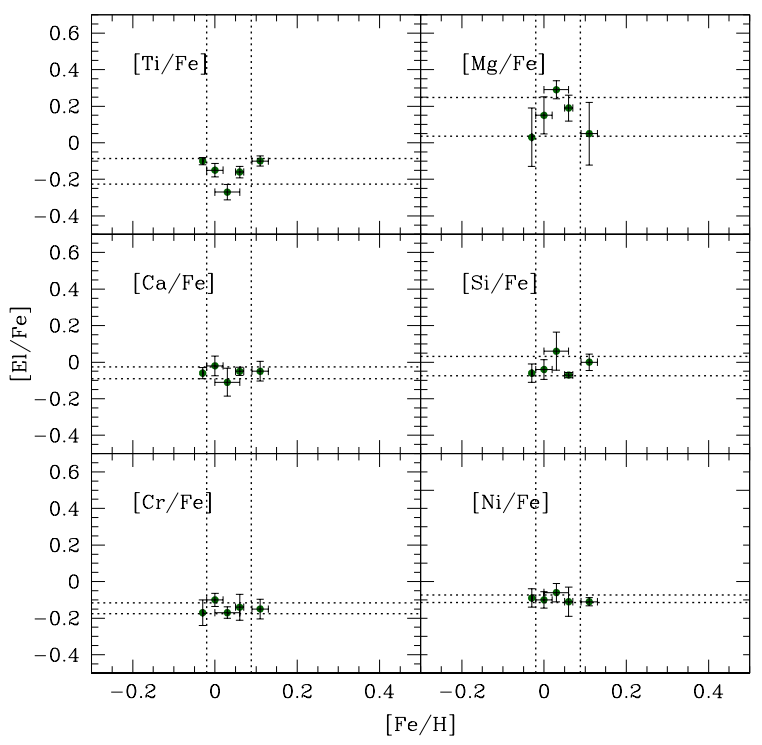

Fig. 4. As in Fig. 3 , but for NGC 4815.

\subsection{A comparison of abundance distributions in the field and clusters}

The uniformity of the analysis of cluster and field stars offers the possibility to see differences in abundances at a level not possible before, when heterogeneous samples and analysis methods were considered. We remind that our comparison is done with stars belonging to different evolutionary stages, such as solar neighbourhood turn-off stars, open cluster clump giants, and inner disk giants. Even though these samples are subjected to a homogeneous analysis, systematic offsets in abundances could arise. Indeed, as a matter of caution when comparing stars in different evolutionary stages, we shall recall a recent analysis of stellar spectra for stars in the open cluster M 67. Önehag et al. (2011) analysed a solar twin in M 67, a dwarf star with stellar parameters very similar to those of the Sun, and found an $[\mathrm{Fe} / \mathrm{H}]$ ratio of $+0.02 \mathrm{dex}$ and $[\mathrm{El} / \mathrm{Fe}]$ within 0.03 dex of the solar values. This is in contrast to analysis of evolved giant stars in the same cluster for which Yong et al. (2005) found an $[\mathrm{Fe} / \mathrm{H}]$ ratio very close to the solar value but with abundance ratios that differ significantly from the solar values. However, we consider that, more than the difference in evolutionary stages, different methods and different atomic data might affect the results of the analysis of Önehag et al. (2011) and of Yong et al. (2005). A contrasting example can be found in the study of IC 4615 (Pasquini et al. 2004) who analysed both dwarf and giant stars, for which no differences in the chemical composition were found.

As also pointed by Meléndez et al. (2008), the use of the same set of lines and the choice of a common solar abundance 


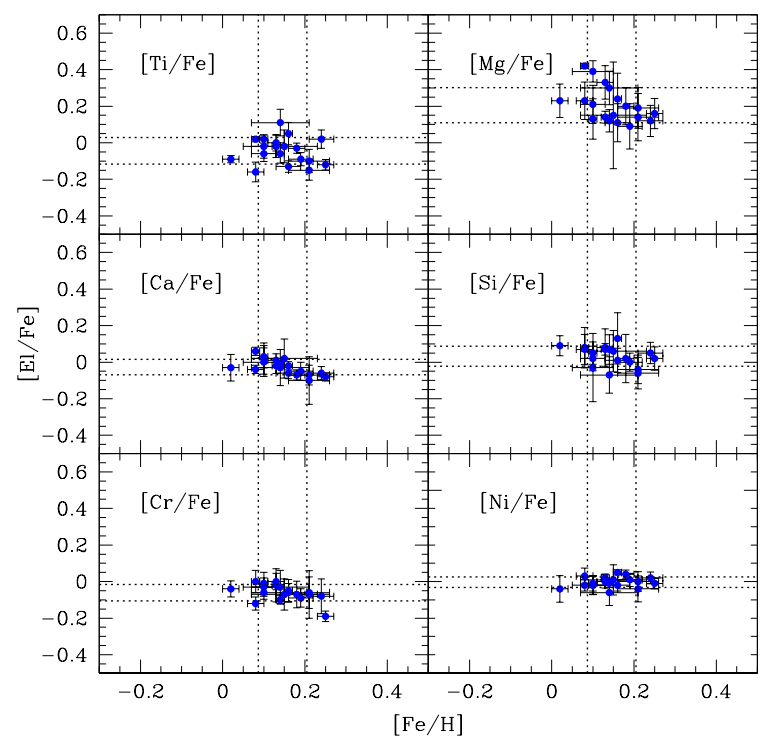

Fig. 5. As in Fig. 3 , but for NGC 6705.

scale for the normalisation of the stellar results are of great importance, and are among the main strengths of our analysis. We recall that different conclusions were drawn for instance from a comparison of bulge and thick disk stars in Fulbright et al. (2007) and Meléndez et al. (2008). Those differences are probably due to the heterogeneous comparison in the former paper of their bulge giant results with literature values for main sequence and turn-off disk stars in the solar neighbourhood (Bensby et al. 2005; Reddy et al. 2006) analysed with a different line list and normalised to a different solar zero-point. In the Gaia-ESO survey analysis, all stars are analysed as homogeneously as possible, and these systematic effects should be reduced.

In Fig. 8 we show the abundance ratios $[\mathrm{El} / \mathrm{Fe}]$ versus $[\mathrm{Fe} / \mathrm{H}]$ of solar neighbourhood dwarf stars, of inner disk/bulge giant stars and of clusters. We note that elemental abundances of stars in open clusters are consistent, within the errors, with the trends of $[\mathrm{El} / \mathrm{Fe}]$ versus $[\mathrm{Fe} / \mathrm{H}]$ for almost all elements in field stars. However cluster stars show some differences from field stars having the same $[\mathrm{Fe} / \mathrm{H}]$.

A statistical way to compare two distinct populations is to compare their cumulative distributions. In our case we want to probe possible differences in the chemical composition of two populations, thus we compare the cumulative distribution of their elemental abundance ratios. For each cluster we have selected for the comparison only field stars (both solar neighbourhood and inner-disk/bulge stars) in the same metallicity range, $0.2 \mathrm{dex}$, centred around the mean metallicity of the cluster stars: $0.05 \leq[\mathrm{Fe} / \mathrm{H}]<0.25$ for Trumpler $20,0.0 \leq[\mathrm{Fe} / \mathrm{H}]<0.2$ for NGC6705 and $-0.1 \leq[\mathrm{Fe} / \mathrm{H}]<0.1$ for NGC 4815 . The cumu-

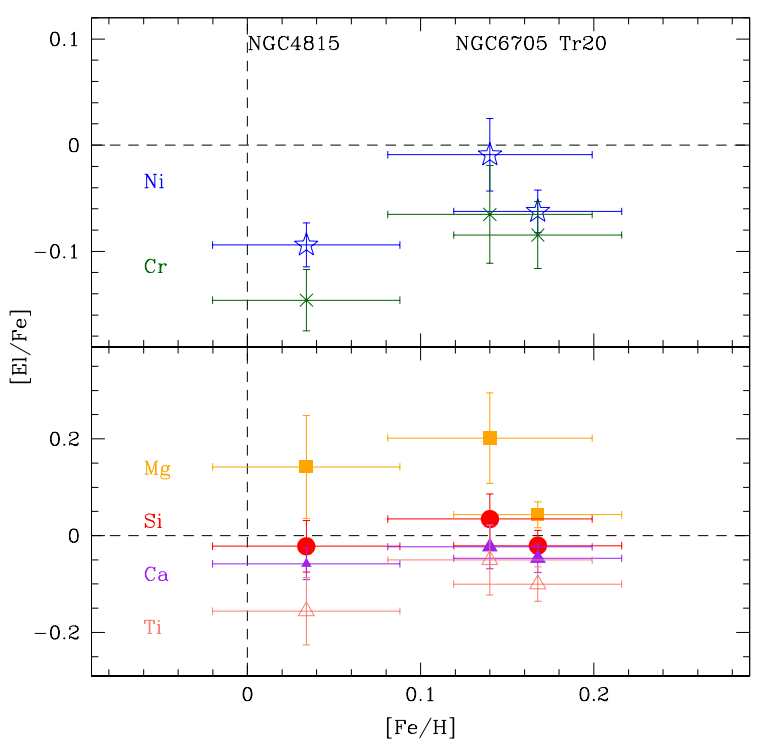

Fig. 6. Average values of the four $\alpha$-elements $(\mathrm{Ca}, \mathrm{Si}, \mathrm{Ti}, \mathrm{Mg})$ in the bottom panel. Results of iron-peak elements $(\mathrm{Ni}, \mathrm{Cr})$ are shown in the upper panels. Clusters are ordered by $[\mathrm{Fe} / \mathrm{H}], \mathrm{NGC}$ 4815 is the first cluster on the left, NGC 6705 in the middle, and Trumpler 20 on the right side. The errors are the standard deviation, $\sigma$, computed for member stars of each clusters. The colour and symbol code is the following: green crosses for $[\mathrm{Cr} / \mathrm{Fe}]$, blue stars for $[\mathrm{Ni} / \mathrm{Fe}]$, red circles for $[\mathrm{Si} / \mathrm{Fe}]$, purple filled triangles for $[\mathrm{Ca} / \mathrm{Fe}]$, orange squares for $[\mathrm{Mg} / \mathrm{Fe}]$, and empty salmon triangles for $[\mathrm{Ti} / \mathrm{Fe}]$.

lative distributions of abundance ratios are shown in Figs. 9, 10 . 11. The closer two distributions are, the higher is the probability that they come from populations sharing the same chemical composition. For instance, $[\mathrm{Ca} / \mathrm{H}]$ distribution in $\operatorname{Tr} 20$ and in the solar neighbourhood stars have a probability of $\sim 60 \%$ to derive from the same population, while for $[\mathrm{Mg} / \mathrm{Fe}]$ the probability is lower than $1 \%$. For NGC 4815 , the highest probabilities of similar distributions are for $[\mathrm{Mg} / \mathrm{Fe}](\sim 90 \%),[\mathrm{Ni} / \mathrm{Fe}]$ ( $\sim 50 \%),[\mathrm{Si} / \mathrm{Fe}](\sim 20 \%)$ in NGC 4815 and in the solar neighbourhood. For NGC 6705 , the probabilities that the cluster and inner-disk stars came from similar populations are: $[\mathrm{Cr} / \mathrm{Fe}]$ ( 30\%), [Ca/Fe] ( 50\%), [Mg/Fe] $(\sim 70 \%),[\mathrm{Ni} / \mathrm{Fe}](\sim 80 \%)$, $[\mathrm{Ti} / \mathrm{Fe}](\sim 10 \%)$. Due to the small number statistics of our analysis we recall that the probabilities associated with the statistical Kolmogorov-Smirnov test have a limited confidence and are only indicative.

In Fig. 9 we have the results for Trumpler 20: this cluster is indistinguishable from the field population in its iron-peak elements. The alpha-elements do not all behave in the same way: two of them are remarkably under-abundant compared to field 


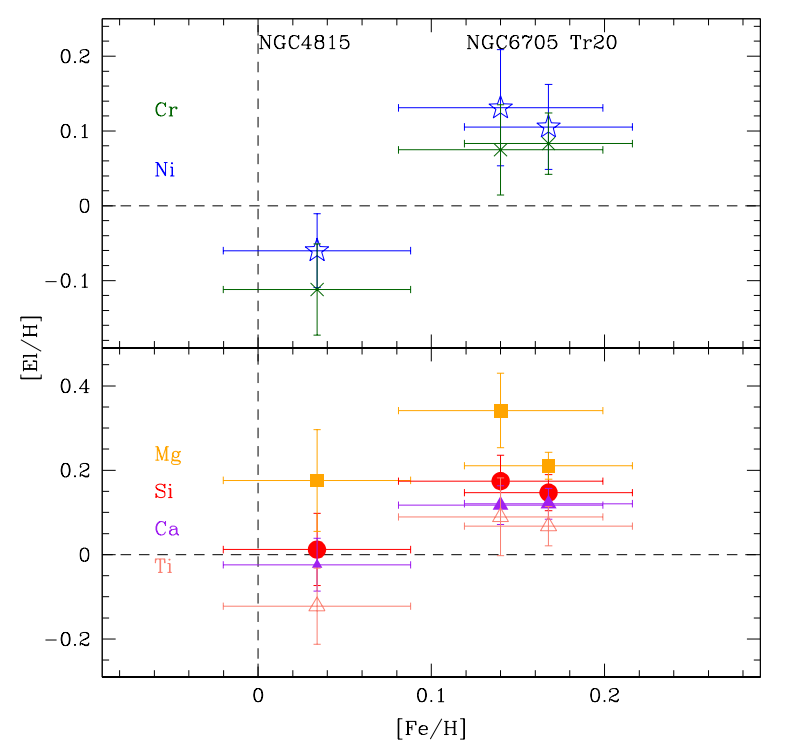

Fig. 7. As in Fig 6 but for $[\mathrm{El} / \mathrm{H}]$.

stars with the same metallicity ( $\mathrm{Mg}$ and $\mathrm{Ti}$ ), while $\mathrm{Ca}$ and $\mathrm{Si}$ are distributed similarly to the inner-disk/bulge stars. In Fig. 10 we show the results for NGC 4815 , which exhibits a clear underabundance of $\mathrm{Ti}$, and has $[\mathrm{Si} / \mathrm{Fe}]$ between the solar neighbourhood and inner disk stars, while $\mathrm{Mg}$ and $\mathrm{Ca}$ are similar to the solar neighbourhood sample. Among the iron-peak elements $\mathrm{Cr}$ is slightly lower than that in the solar neighbourhood sample, while Ni has the same distribution. In Fig. 11 we present the results for NGC 6705: the behaviour of this cluster is very similar to that of stars located in the inner-disk/bulge, having similar distribution of $\mathrm{Mg}, \mathrm{Ti}, \mathrm{Ca}, \mathrm{Cr}$, and $\mathrm{Ni}$. Only $\mathrm{Si}$ is enhanced with respect to both solar neighbourhood and inner-disk stars.

\subsection{Ti abundances in stars of different type}

Titanium is evidently more under-abundant in Trumpler 20 and NGC 4815 than in the field population with the same $[\mathrm{Fe} / \mathrm{H}]$ (see Figs. 9 and 10 . Thus it might be a good tracer of the different abundance patterns of these two populations. To prove that this difference is real and not due to NLTE over-ionisation effects in the considered range of stellar parameters, we have plotted [TiII/FeII]-[TiI/FeI] vs. Teff , as done by D'Orazi \& Randich (2009), and also vs. $\log g$ and $\xi$. In their study of the open clusters IC 2602 and IC 2391, D'Orazi \& Randich (2009) found indeed some trends of $[\mathrm{El} / \mathrm{Fe}]$ vs. $\mathrm{T}_{\text {eff }}$ for various elements. These effects were already found for old field stars by Bodaghee et al. (2003) and by Gilli et al. (2006), who explained them as due to NLTE effects. Other explanations were given from Adibekyan et

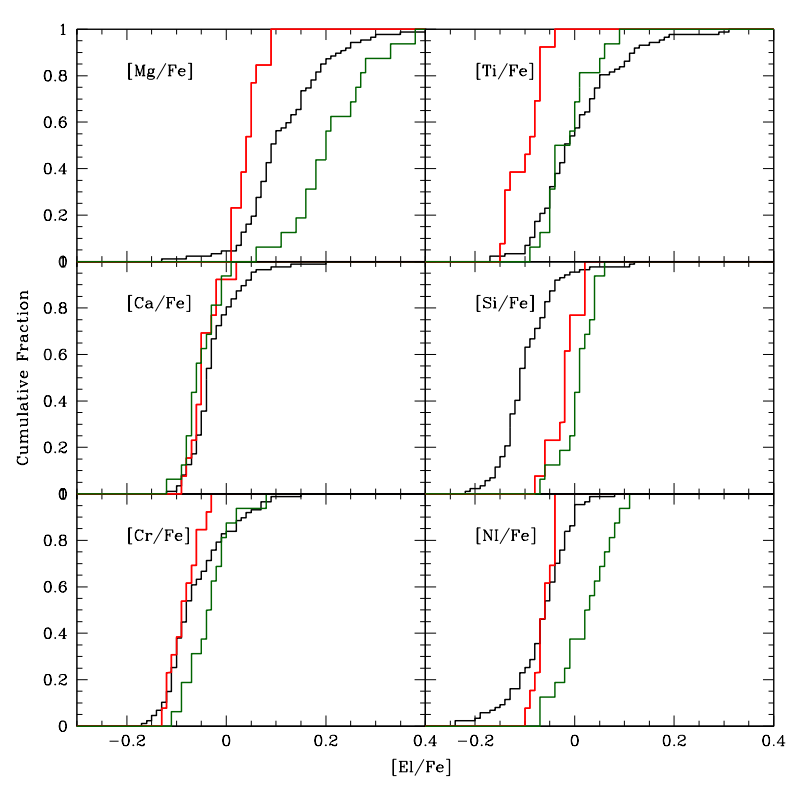

Fig. 9. Comparison of cumulative distribution of the Trumpler 20 abundance ratios (red curves) with the solar neighbourhood turnoff stars with the same metallicity (black curves) and with the inner disk/bulge giant stars (green curves).

al. (2012) who analysed a large sample of F-G-K stars for which they studied the relation between $[\mathrm{Ti} / \mathrm{TiII}]$ and $\mathrm{T}_{\text {eff } f}$. They found a trend that they explained with problems associated with the differential analysis or with an incorrect opacity in the model atmospheres. For Ca and Ti, D'Orazi \& Randich (2009) obtained decreasing trends with temperature, with cooler stars having lower $[\mathrm{Ca} / \mathrm{Fe}]$ and $[\mathrm{Ti} / \mathrm{Fe}]$ than hotter stars in the same cluster. They proposed that the young age of their stars and the enhanced levels of chromospheric activity affect them by NLTE over-ionisation (see their Fig. 4). This trend is completely absent in the three panels of Fig. 12, [TiII/FeII]-[TiI/FeI] is almost consistent with zero along the whole $\mathrm{T}_{e f f}, \log \mathrm{g}$ and $\xi$ ranges. This is likely due to the older age of our stars, and reassures us about the use of titanium as a reliable tracer of the abundance patterns of open clusters. We did also a computation of the NLTE effects on a sample of TiI and TiII lines employed in the analysis of the Gaia-ESO Survey. We found that on average the NLTE correction for TiI are of the order of $+0.06 \mathrm{dex}$, while TiII is not affected by NLTE effects (Bergemann, private communication). 


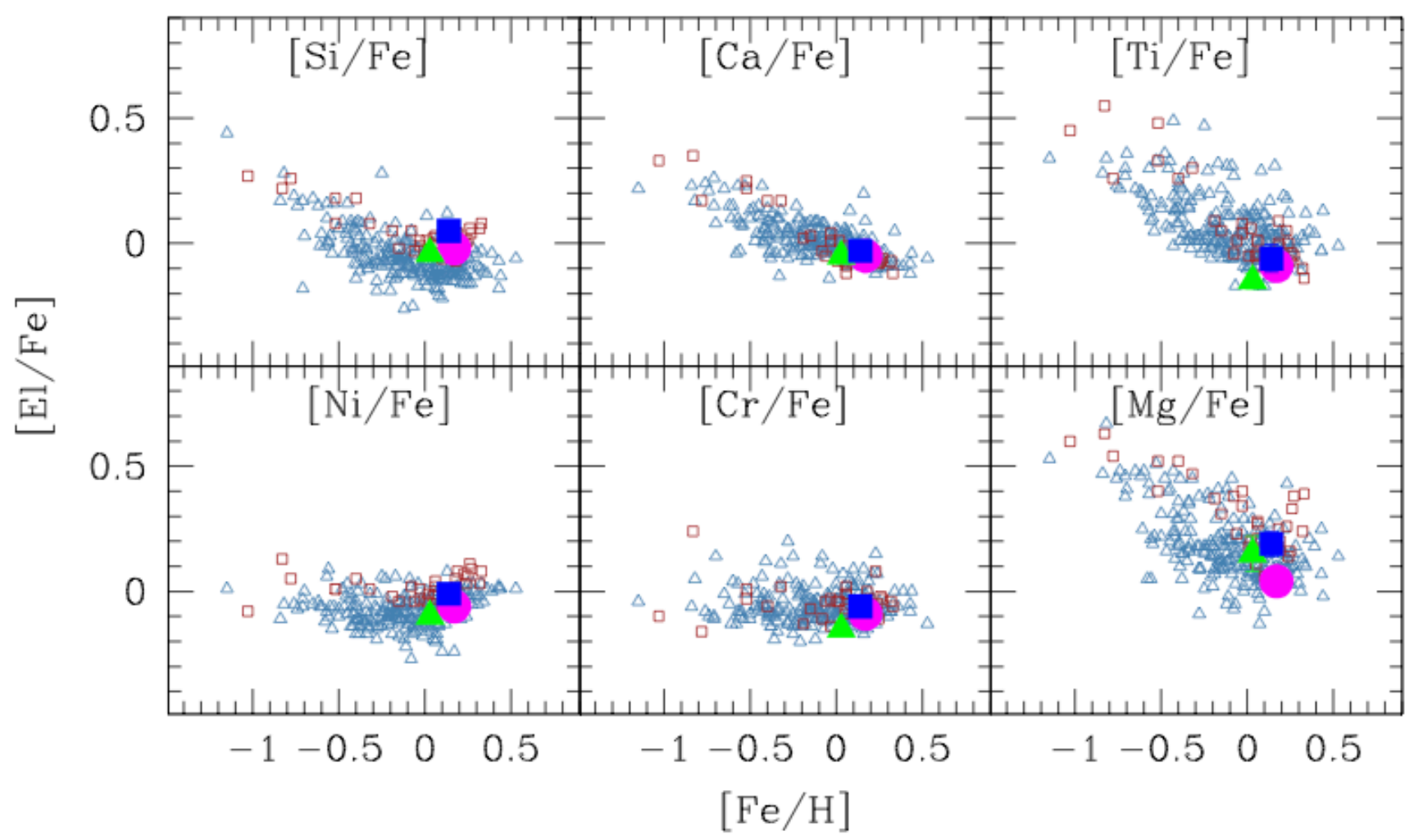

Fig. 8. Abundance ratios in field and open cluster stars: the abundance ratios $[\mathrm{El} / \mathrm{Fe}]$ versus $[\mathrm{Fe} / \mathrm{H}]$ of solar neighbourhood dwarf stars (cyan empty triangles), of inner disk/bulge giant stars (red empty squares). Clusters are represented by their average values: Trumpler 20 (magenta filled circle), NGC 4815 (green filled triangle), and NGC 6705 (blue filled square).

\section{On the origin of the abundance ratios in the inner open clusters}

In this section we discuss what we can learn about the origin of the inner disk open clusters from their abundance patterns. We discuss only elements which are not affected by stellar evolution, and thus direct tracers of the ISM composition at the epoch when the cluster formed: the $\alpha$ - and iron-peak elements. We compare their abundance ratios in the field and cluster populations with the predictions of two different chemical evolution models (Magrini et al. 2009, Romano et al. 2010, hereafter M09 and R10, respectively), briefly described below.

Model of Romano et al. (2010) The model of R10 is based on the two-infall model case $B$ for the chemical evolution of the Galaxy. A thorough discussion of the adopted formalism and basic equations can be found in Chiappini et al. (1997, 2001). Here we briefly recall the overall evolutionary scenario. The inner halo and thick disk of the Milky Way are assumed to form on a relatively short timescale (about $1 \mathrm{Gyr}$ ) out of a first infall episode, whereas the thin disk forms inside-out (Matteucci \& Francois 1989) on longer timescales (7 Gyr in the solar vicinity) during a second independent episode of extragalactic gas infall. The Galactic disk is approximated by several independent rings, $2 \mathrm{kpc}$ wide. Radial flows and outflows are not considered here.
The adopted star formation rate (SFR) is proportional to both the total mass and the gas surface densities. The efficiency of conversion of gas into stars is higher during the halo/thick-disk phase than during the thin-disk phase. Furthermore, it drops to zero every time the gas density drops below a critical density threshold. The stellar lifetimes are taken into account in detail. As for the stellar IMF, the Kroupa et al. (1993) IMF is assumed in the $0.1-100 \mathrm{M}_{\odot}$ mass range. The rate of SNIa explosions is calculated as in Matteucci \& Greggio (1986). SNeIa explode in close binary systems when a $\mathrm{CO}$ white dwarf has reached a critical mass limit because of accretion of hydrogen-rich matter from a main-sequence or red giant companion. The yields for SNeIa are taken from Iwamoto et al. (1999), model W7. As for single stars, several sets of stellar yields are analysed by R10 (see their table 2). Here we show the results of their model 15, that adopts the yields by Karakas (2010) for low- and intermediatemass stars $\left(1-6 \mathrm{M}_{\odot}\right)$, the yields of rotating massive stars by the Geneva group (see R10, their table 1 for references) for helium, carbon, nitrogen and oxygen, and the yields of Kobayashi et al. (2006) for heavier elements produced by Type II supernovae and hypernovae with progenitor masses in the range $13-40 \mathrm{M}_{\odot}$. The available stellar yields are interpolated in the mass range 6$13 \mathrm{M}_{\odot}$ and extrapolated up to $100 \mathrm{M}_{\odot}$ (see R10 for details). The tabulated yields are adopted as published, i.e. without ad hoc adjustments to reproduce the data. This notwithstanding, model 15 


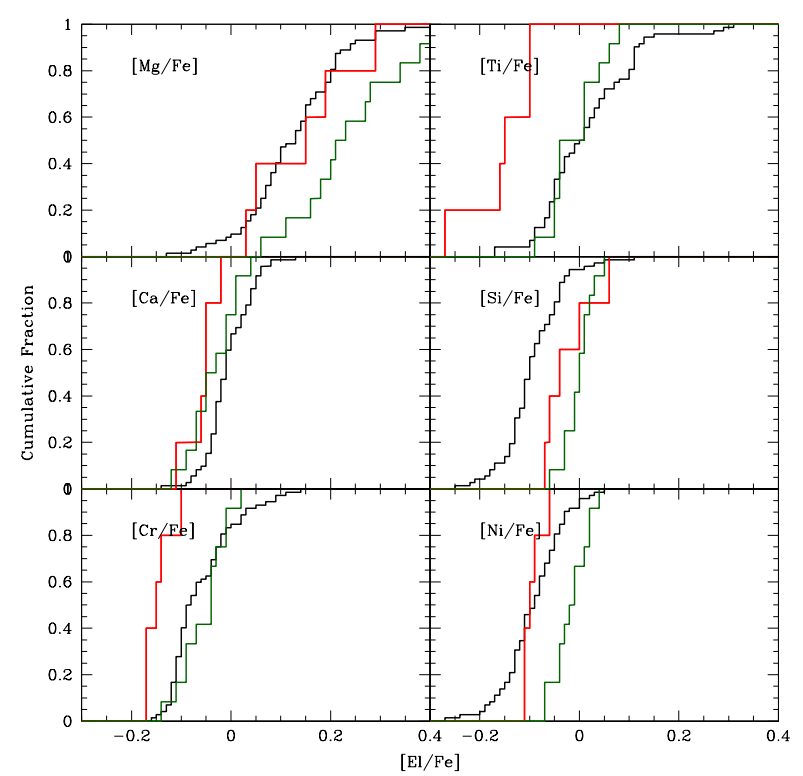

Fig. 10. As in Figure 9 , but for NGC 4815.

provides a good fit to the abundance ratios of most chemical elements in Milky Way's stars.

Model of Magrini et al. 2009 The model adopted by M09 is a generalisation of the multi-phase model by Ferrini et al. (1992), originally built for the solar neighbourhood, and subsequently extended to the entire Galaxy (Ferrini et al. 1994) and to other disk galaxies (e.g., Mollá et al. 1996, Mollá \& Díaz 2005, Magrini et al. 2007). The detailed description of the general model is in the above mentioned papers. The main assumptions of the model are that the Galaxy disk is formed by infall of gas from the halo and from the intergalactic medium. The adopted infall follows an exponentially decreasing law. This produces an inside-out formation scenario where the inner parts of the disk evolve more rapidly than the outer ones. As in the R10 model, radial flows and stellar migrations, as well as gas outflows are not considered. The stellar lifetimes are taken into account in details and for the stellar yields the following choices were made: for low- and intermediate-mass stars $\left(\mathrm{M}<8 \quad M_{\odot}\right)$ they use the yields by Gavilán et al. (2005) for both values of the metallicity $(\mathrm{Z}=0.006$ and $\mathrm{Z}=0.02)$. For stars in the mass range $8 M_{\odot}<M<$ $35 M_{\odot}$ they adopted the yields by Chieffi \& Limongi (2002) for $\mathrm{Z}=0.006$ and $\mathrm{Z}=0.02$. They estimated the yields of stars in the mass range $35 M_{\odot}<M<100 M_{\odot}$, which are not included in the tables of Chieffi \& Limongi (2004), by linear extrapolation of the yields in the mass range $8 M_{\odot}<M<35 M_{\odot}$. In the case

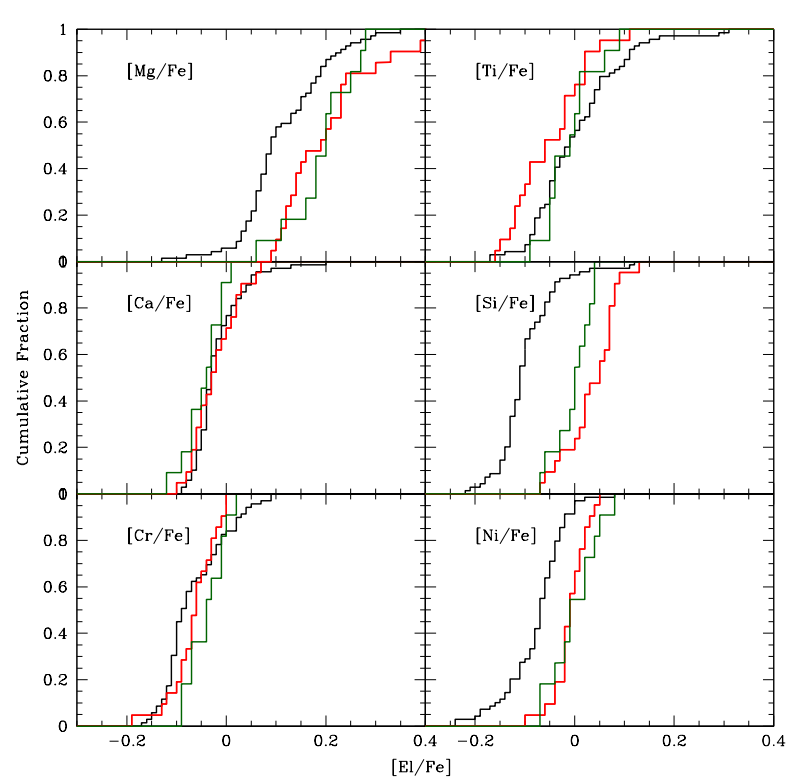

Fig. 11. As in Figure 9 , but for NGC 6705.

of Ti, M09 increased the yields by a factor of 2 to reproduce the observations. The rate of SNIa explosions is calculated as in Matteucci \& Greggio (1986) and their yields are taken from the model CDD1 by Iwamoto et al. (1999). The IMF by Kroupa et al. (1993) is adopted.

Comparison models vs data In Fig. 13 we show the results of the two models: R10 in panel a) and M09 in panel b). The curves refer to different Galactic radii, $4 \mathrm{kpc}, 6 \mathrm{kpc}$ and $8 \mathrm{kpc}$. Both models are those originally published by R10 and M09, and are not modified to reproduce the Gaia-ESO Survey data. Thus the agreement with the data, and the similarity of their predictions for the Solar radius are encouraging, even if there are some discrepant elements. The only exception is $\mathrm{Ni}$ for which different yields are adopted by R10 and M09. We note also that the stellar yields adopted in both models are not able to reproduce the trends of $[\mathrm{Ti} / \mathrm{Fe}]$ vs $[\mathrm{Fe} / \mathrm{H}]$, as already noticed in the original papers.

Notwithstanding the agreement at the Solar radius, the two models show a different behaviour in the inner disk, where different assumptions of the infall and star formation rates are made. This might be ascribed to the paucity of observational constraints in the inner disk when the two models were designed. We note indeed that in panel a), the model curves reach higher metallicities (by $\sim 0.2 \mathrm{dex}$ ) at the innermost radius because of the more intense SFR, but the predicted $[\mathrm{El} / \mathrm{Fe}]$ ratios are pretty much the 
same. In panel $\mathrm{b}$ ), the evolution of the $\alpha$ - and iron-peak elements behaves differently with respect to the Galactocentric radius: while the iron-peak elements are quite insensitive to the choice of the Galactocentric radius, the $\alpha$-elements are more enhanced in stars born at $\mathrm{R} \sim 4 \mathrm{kpc}$ than at the Solar radius. This can be easily explained with the short time scales for the gas consumption in the inner part of the disk due to the high star formation rate, which typically produce high $\alpha$ over iron abundances. On the other hand, the iron-peak elements are insensitive to the radius since they behave, to first order, as iron.

For some $\alpha$-elements, $\mathrm{Si}$ and $\mathrm{Mg}$, and marginally also $\mathrm{Ca}$ and Ti, both the inner-disk/bulge stars and NGC 6705 are in better agreement with the curves corresponding to the innermost radii of M09, i.e. in between 4 and $6 \mathrm{kpc}$ from the Galactic Center. On the other hand, the $\alpha$ abundance ratios of Trumpler 20 and of NGC 4815 agree better with the curve corresponding to their present radius in M09 and with the curves of R10. For the ironpeak elements the behaviour of the three clusters is very similar and in agreement with the model curves, of both M09 and R10, which do not predict strong variation of these ratios with the Galactocentric radii.

The comparison of the abundance ratios with the model curves together with the similarity observed between the cumulative distributions of abundance ratios and of the solar neighbourhood and inner-disk/bulge stars, gives us a "chemical" indication of the birthplace of the three inner-disk clusters under analysis: the good agreement of the $[\alpha / \mathrm{Fe}]$ abundance ratio of Trumpler 20 and NGC 4815 with the curves at $\sim 6-8 \mathrm{kpc}$ of both models, and at the same time with the abundance ratios of solar neighbourhood stars, is a reasonable indication that they were born not very far from their present location. On the other hand, the good agreement within the error of some abundance ratios of NGC 6705 with M09 curves for radii 4-6 kpc, and with the observations of inner-disk/bulge stars (see Fig. 111), might indicate that it has moved towards its present position from an inner birthplace. This is in agreement with the orbit determination done for this cluster in Magrini et al. (2010) with a perigalacticon of $\sim 5 \mathrm{kpc}$ and apogalacticon of $\sim 9 \mathrm{kpc}$. Under this scenario, a revision of the basic assumptions of the model of R10 will be required for the model to be able to predict higher SFRs/steeper abundance gradients in the inner disk and to face the Gaia-ESO Survey abundance data for those clusters. For instance, the inclusion of a bar in the model could lead to enhanced star formation in the inner Galactic region (see, e.g., Wang et al. 2012). In addition, the effect of local inhomogeneities, radial flows, stellar migration, and outflow should not be neglected in next generation of chemical evolution models.

\section{Summary}

In this paper we present the analysis of abundance ratios in open clusters and field stars obtained in the first six months of the Gaia-ESO Survey. We studied three old/intermediate-age open clusters: NGC 6705, NGC 4815, and Trumpler 20. For the three clusters we find that: $i$ ) the clusters are internally homogeneous in the considered elements (four $\alpha$-elements, $\mathrm{Si}, \mathrm{Ca}, \mathrm{Mg}$, Ti and three iron-peak elements, $\mathrm{Fe}, \mathrm{Ni}, \mathrm{Cr}$ ); ii) the three clusters have similar $[\mathrm{El} / \mathrm{Fe}]$ abundance patterns, but different global metallicity and, consequently, different $[\mathrm{El} / \mathrm{H}]$ patterns; iii) a comparison of the cumulative distributions of abundance ratios shows that the abundance ratios of NGC 6705 are very similar to those of inner-disk/bulge stars studied by the Gaia-ESO Survey, while the abundance patterns of NGC 4815 and Trumpler 20 do not match perfectly either with the solar neighbourhood stars or with the inner-disk/bulge stars. We finally compare the field and cluster abundance ratios with two chemical evolution models (M09 and R10), finding a general good agreement for the solar neighbourhood. The predictions of the models differ for the inner disk. The $\alpha$-enhancement of NGC 6705 places it in better agreement with the model curves of M09 for Galactocentric radii from 4 to $6 \mathrm{kpc}$, and, together with its better agreement with the distributions of abundances ratios in the inner-disk/bulge sample, supports an inner birthplace for it. Concluding, the first results from the Gaia-ESO Survey show its huge potential to give new constraints to our view of the Galactic chemical evolution, to explore areas of our Galaxy so far little studied, and moreover to put, for the first time, many stellar populations on exactly the same scale.

Acknowledgements. We acknowledge the support from INAF and Ministero dell'Istruzione, dell'Universitá e della Ricerca (MIUR) in the form of the grant "Premiale VLT 2012". The results presented here benefited from discussions in three Gaia-ESO workshops supported by the ESF (European Science Foundation) through the GREAT (Gaia Research for European Astronomy Training) Research Network Program (Science meetings 3855, 4127 and 4415) This work was partially supported by the Gaia Research for European Astronomy Training (GREAT-ITN) Marie Curie network, funded through the European Union Seventh Framework Programme [FP7/2007-2013] under grant agreement n. 264895. T.B. was funded by grant No. 621-2009-3911 from The Swedish Research Council. This work was partly supported by the European Union FP7 programme through ERC grant number 320360. This work was partly supported by the Leverhulme Trust through grant RPG-2012-541. I.S.R. gratefully acknowledges the support provided by the Gemini-CONICYT project 32110029. This research has made use of the SIMBAD database, operated at CDS, Strasbourg, France.

\section{References}

Adibekyan, V. Z., Sousa, S. G., Santos, N. C., et al. 2012, A\&A, 545, A32

Allende Prieto, C., Majewski, S. R., Schiavon, R., et al. 2008, Astronomische Nachrichten, 329, 1018

Andreuzzi, G., Bragaglia, A., Tosi, M., \& Marconi, G. 2011, MNRAS, 412, 1265 Andrievsky, S. M., Spite, M., Korotin, S. A., et al. 2007, A\&A, 464, 1081

Baglin, A., Auvergne, M., Boisnard, L., et al. 2006, 36th COSPAR Scientific Assembly, 36, 3749

Bensby, T., Feltzing, S., Lundström, I., \& Ilyin, I. 2005, A\&A, 433, 185

Bodaghee, A., Santos, N. C., Israelian, G., \& Mayor, M. 2003, A\&A, 404, 715

Bonatto, C., Kerber, L. O., Bica, E., \& Santiago, B. X. 2006, A\&A, 446, 121

Bragaglia, A., \& Tosi, M. 2006, AJ, 131, 1544

Carraro, G., Ng, Y. K., \& Portinari, L. 1998, MNRAS, 296, 1045

Chen, L., Hou, J. L., \& Wang, J. J. 2003, AJ, 125, 1397

Chiappini, C., Matteucci, F., \& Romano, D. 2001, ApJ, 554, 1044

Chiappini, C., Matteucci, F., \& Gratton, R. 1997, ApJ, 477, 765

Chieffi, A., \& Limongi, M. 2002, ApJ, 577, 281

Dekker, H., D’Odorico, S., Kaufer, A., Delabre, B., \& Kotzlowski, H. 2000, Proc. SPIE, 4008, 534

D’Orazi, V., \& Randich, S. 2009, A\&A, 501, 553

de Laverny, P., Recio-Blanco, A., Worley, C. C., \& Plez, B. 2012, A\&A, 544, A126

De Silva, G. M., Freeman, K. C., Asplund, M., et al. 2007, AJ, 133, 1161

Ferrini, F., Matteucci, F., Pardi, C., \& Penco, U. 1992, ApJ, 387, 138

Ferrini, F., Molla, M., Pardi, M. C., \& Diaz, A. I. 1994, ApJ, 427, 745

Freeman, K. C. 1970, ApJ, 160, 811

Friel, E. D. 1995, ARA\&A, 33, 381

Frinchaboy, P. M., Thompson, B., Jackson, K. M., et al. 2013, arXiv:1308.4195

Fulbright, J. P., McWilliam, A., \& Rich, R. M. 2007, ApJ, 661, 1152

Gavilán, M., Buell, J. F., \& Mollá, M. 2005, A\&A, 432, 861

Gieles, M., Portegies Zwart, S. F., Baumgardt, H., et al. 2006, MNRAS, 371, 793

Gilli, G., Israelian, G., Ecuvillon, A., Santos, N. C., \& Mayor, M. 2006, A\&A, 449,723

Gilmore, G., Randich, S., Asplund, M., et al. 2012, The Messenger, 147, 25 Grevesse, N., Asplund, M., \& Sauval, A. J. 2007, Space Sci. Rev., 130, 105 Gustafsson, B., Edvardsson, B., Eriksson, K., et al. 2008, A\&A, 486, 951 Jacobson, H. R., Pilachowski, C. A., \& Friel, E. D. 2011, AJ, 142, 59 
Janes, K. A. 1979, ApJS, 39, 135

Janes, K. A., \& Phelps, R. L. 1994, AJ, 108, 1773

Jofre, P., Heiter, U., Blanco-Cuaresma, S., \& Soubiran, C. 2013 , arXiv:1312.2943

Jofre, P., Heiter, U., Soubiran, C., et al. 2013, arXiv:1309.1099

Karakas, A. I. 2010, MNRAS, 403, 1413

Kobayashi, C., Umeda, H., Nomoto, K., Tominaga, N., \& Ohkubo, T. 2006, ApJ, 653,1145

Kroupa, P., Tout, C. A., \& Gilmore, G. 1993, MNRAS, 262, 545

Iwamoto, K., Brachwitz, F., Nomoto, K., et al. 1999, ApJS, 125, 439

Lada, C. J., \& Lada, E. A. 2003, ARA\&A, 41, 57

Magrini, L., Corbelli, E., \& Galli, D. 2007, A\&A, 470, 843

Magrini, L., Sestito, P., Randich, S., \& Galli, D. 2009, A\&A, 494, 95

Magrini, L., Randich, S., Zoccali, M., et al. 2010, A\&A, 523, A11

Matteucci, F., \& Francois, P. 1989, MNRAS, 239, 885

Matteucci, F., \& Greggio, L. 1986, A\&A, 154, 279

McMahon, R. 2012, Science from the Next Generation Imaging and Spectroscopic Surveys,

Meléndez, J., Asplund, M., Alves-Brito, A., et al. 2008, A\&A, 484, L21

Mermilliod, J.-C. 1996, The Origins, Evolution, and Destinies of Binary Stars in Clusters, 90, 475

Mermilliod, J.-C., Mayor, M., \& Udry, S. 2009, A\&A, 498, 949

Mollá, M., Ferrini, F., \& Diaz, A. I. 1996, ApJ, 466, 668

Mollá, M., \& Díaz, A. I. 2005, MNRAS, 358, 521

Nordström, B., Mayor, M., Andersen, J., et al. 2004, A\&A, 418, 989

Önehag, A., Korn, A., Gustafsson, B., Stempels, E., \& Vandenberg, D. A. 2011 A\&A, 528, A85

Panagia, N., \& Tosi, M. 1981, A\&A, 96, 306

Pancino, E., Carrera, R., Rossetti, E., \& Gallart, C. 2010, A\&A, 511, A56

Pasquini, L., Avila, G., Blecha, A., et al. 2002, The Messenger, 110,

Pasquini, L., Randich, S., Zoccali, M., et al. 2004, A\&A, 424, 951

Phelps, R. L., Janes, K. A., \& Montgomery, K. A. 1994, AJ, 107, 1079

Randich, S., \& Gilmore, G. 2012, Science from the Next Generation Imaging and Spectroscopic Surveys,

Reddy, B. E., Lambert, D. L., \& Allende Prieto, C. 2006, MNRAS, 367, 1329

Twarog, B. A., Ashman, K. M., \& Anthony-Twarog, B. J. 1997, AJ, 114, 2556

Robin, A. C., Reylé, C., Derrière, S., \& Picaud, S. 2003, A\&A, 409, 523

Rocha-Pinto, H. J., Flynn, C., Scalo, J., et al. 2004, A\&A, 423, 517

Romano, D., Karakas, A. I., Tosi, M., \& Matteucci, F. 2010, A\&A, 522, A32

Santos, J. F. C., Jr., Bonatto, C., \& Bica, E. 2005, A\&A, 442, 201

Scott, J. E., Friel, E. D., \& Janes, K. A. 1995, AJ, 109, 1706

Sestito, P., Bragaglia, A., Randich, S., et al. 2006, A\&A, 458, 121

Sestito, P., Randich, S., \& Bragaglia, A. 2007, A\&A, 465, 185

Sestito, P., Bragaglia, A., Randich, S., et al. 2008, A\&A, 488, 943

Skrutskie, M. F., Cutri, R. M., Stiening, R., et al. 2006, AJ, 131, 1163

van der Kruit, P. C. 2002, The Dynamics, Structure \& History of Galaxies: A Workshop in Honour of Professor Ken Freeman (ASP Conf. Proc. 273), ed. G.S. Da Costa \& H. Jerjen (San Francisco, CA: ASP), 7

Wang, J., Kauffmann, G., Overzier, R., et al. 2012, MNRAS, 423, 3486

Wu, Z.-Y., Zhou, X., Ma, J., \& Du, C.-H. 2009, MNRAS, 399, 2146

Yong, D., Carney, B. W., \& Teixera de Almeida, M. L. 2005, AJ, 130, 597

Yong, D., Carney, B. W., \& Friel, E. D. 2012, AJ, 144, 95 


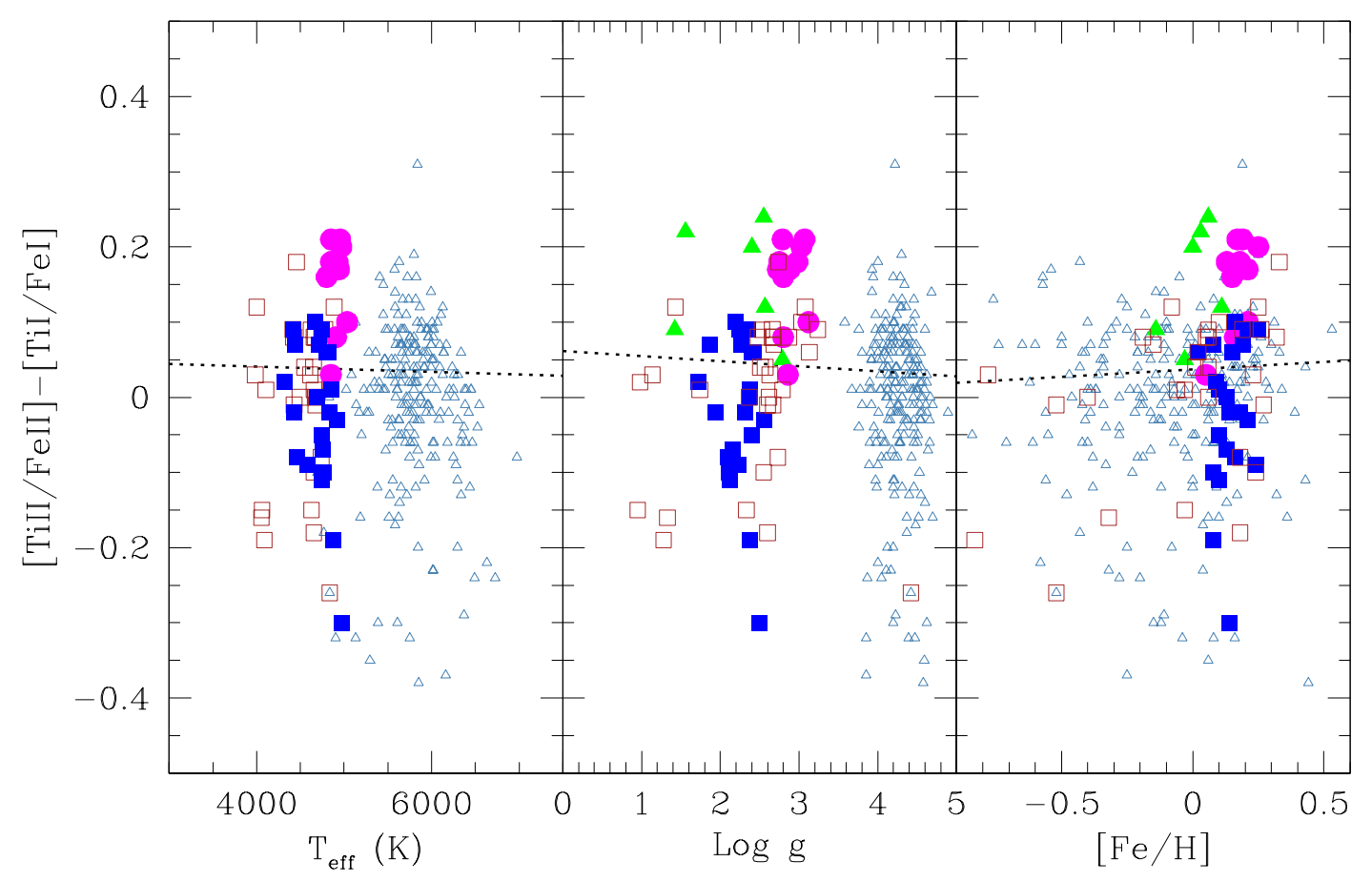

Fig. 12. $[\mathrm{TiII} / \mathrm{Fe}]-[\mathrm{TiI} / \mathrm{Fe}]$ vs. $\mathrm{T}_{e f f}, \log \mathrm{g}$, and $[\mathrm{Fe} / \mathrm{H}]$ in the Milky Way field stars and in the cluster member stars. Symbols as in Fig. 8. The dotted lines are the weighted least mean square fits to the data. 


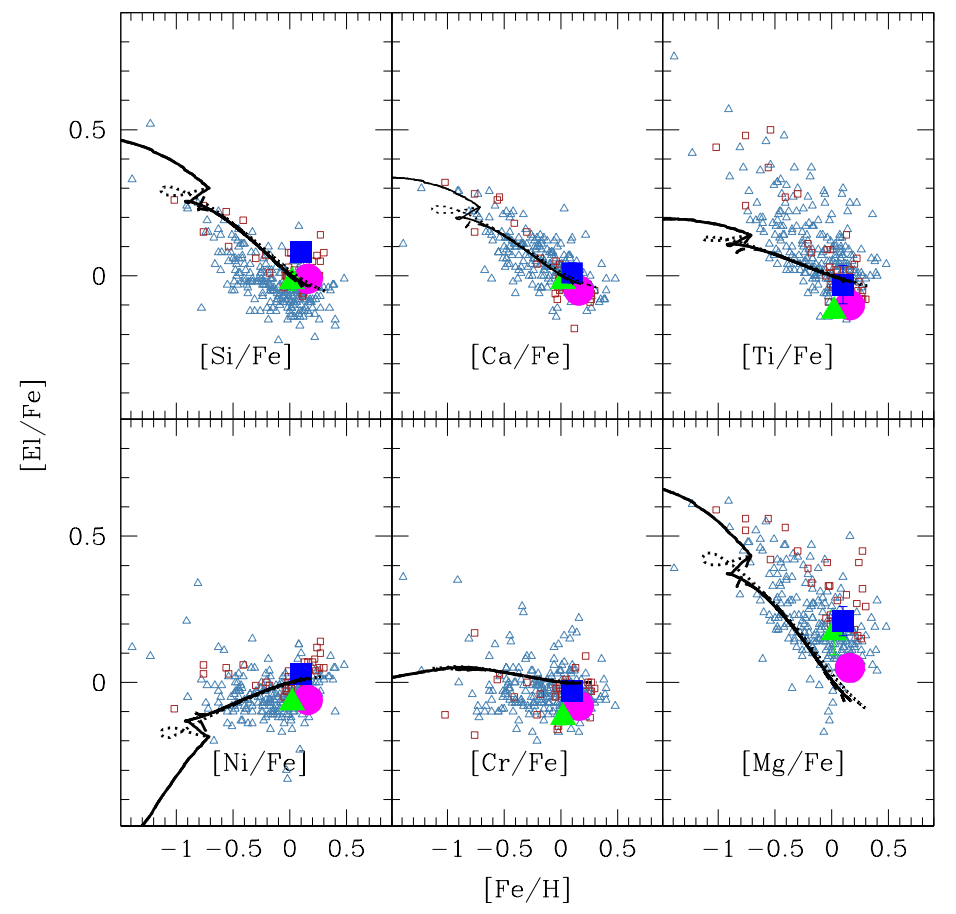

(a) R10

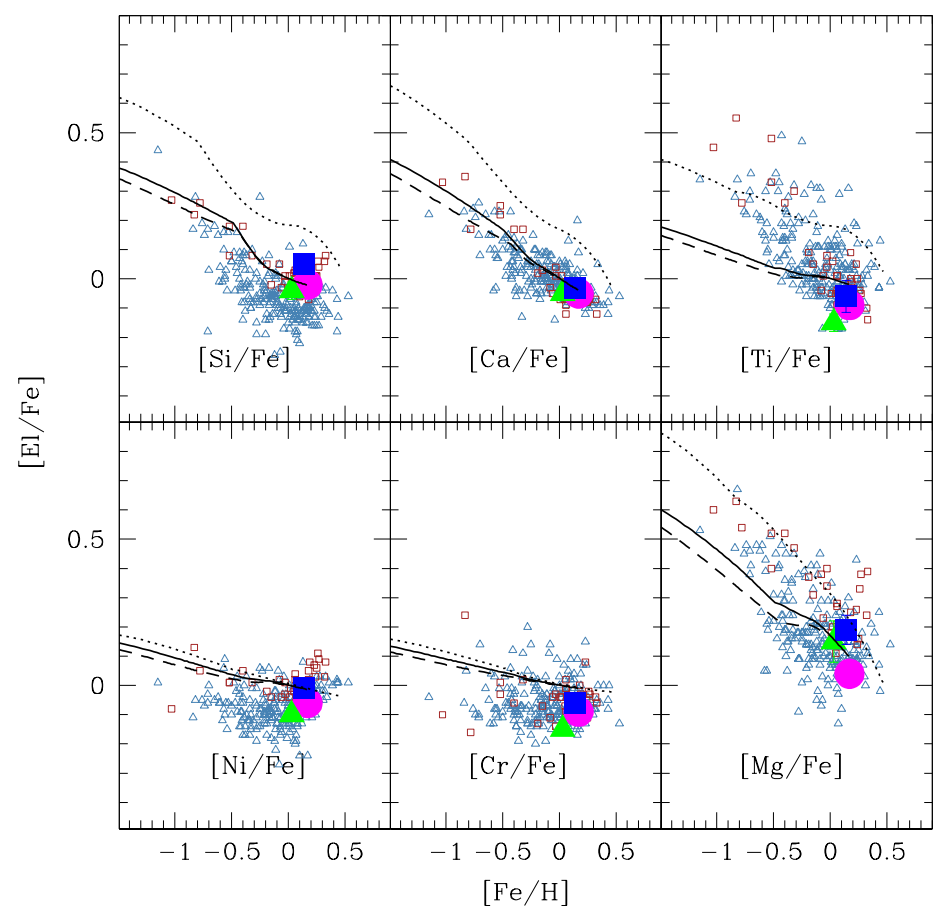

